\title{
Wie lässt sich Verdrängung verhindern?
}

\author{
Die Rent-Gap-Theorie der Gentrifizierung und ihre Gültigkeitsbedingungen am \\ Beispiel des Frankfurter Gallus
}

\author{
Sebastian Schipper \\ Tabea Latocha
}

\begin{abstract}
Innerhalb der Gentrifizierungsforschung analysiert die Rent Gap Theorie, wie kleinräumige Differenzen zwischen gegenwärtigen Verwertungsbedingungen einerseits sowie Erwartungen auf zukünftig steigende Mieten andererseits Verdrängungsprozesse antreiben. Dementgegen hat Eric Clark (2014) jüngst eingefordert, dass die Stadtforschung den Blick verstärkt darauf richten müsse, wie Verdrängung verhindert werden kann. Diesen Appell aufgreifend, zeigen wir bezogen auf den deutschen Kontext, inwiefern mietrechtliche Regularien, stadtplanerische Entscheidungen und die jeweilige Eigentümerstruktur wesentlich darüber entscheiden, ob sich ein immobilienwirtschaftlicher Verwertungsdruck auch tatsächlich in Verdrängungsprozesse übersetzt. Illustriert wird dies am Wandel der Eigentümerstruktur im Frankfurter Gallus seit den 1970er Jahren. Deutlich wird dabei, dass Gentrifizierung kein Naturgesetz darstellt, sondern ein zutiefst politischer Prozess ist, der sich effektiv verhindern lässt.
\end{abstract}

Ersteinreichung: 10. Juni 2017; Veröffentlichung online: 20. Mai 2018

An english abstract can be found at the end of the document.

\section{Einleitung}

Innerhalb der Gentrifizierungsforschung wird mit Hilfe der sogenannten Ertragslücken- bzw. Rent-Gap-Theorie analysiert, inwiefern kleinräumige Differenzen zwischen gegenwärtigen Verwertungsbedingungen einerseits und Erwartungen zukünftig steigender Mieten und Bodenpreise andererseits Aufwertungs- und Verdrängungsprozesse antreiben (Slater 2017; Smith 1996; Smith 1979). Das Erkenntnisinteresse ist dementsprechend primär gerichtet auf die Ursachen städtischer Restrukturierungsprozesse. In seinem 2014 erschienenen Essay fordert dagegen Eric Clark, der im Verlauf seiner akademischen Karriere die Rent-Gap-Theorie maßgeblich geprägt und weiterentwickelt hat (Clark 1995), einen Perspektivwechsel bzw. eine partielle Neuausrichtung der Gentrifizierungsforschung ein. Anstatt in erster Linie darauf zu fokussieren, wie mit Hilfe der Ertragslückentheorie Gentrifizierungsprozesse erklärt werden können, müsse die polit-ökonomische Stadtforschung zukünftig vielmehr den Blick verstärkt darauf richten, unter welchen Bedingungen die Theorie keine Gültigkeit beanspruchen kann. Sein Forschungsauftrag - „make rent gap theory untrue“ (Clark 2014: 394) - zielt somit darauf ab, zu analysieren, wie die politischen 
Rahmenbedingungen in unterschiedlichen Kontexten gestaltet sein müssten, so dass einkommensschwache Schichten trotz Aufwertungsdruck vor Verdrängung geschützt sind. Im Sinne einer angewandten kritischen Geographie könnten eine breitere Öffentlichkeit sowie insbesondere städtische soziale Bewegungen und Mieterinitiativen von einer derartigen Wissensproduktion mit transformatorischem Anspruch profitieren (Slater 2017).

Da sein Essay als Apell formuliert ist und selbst noch kaum Antworten auf die aufgeworfene Problemstellung bietet, wollen wir mit diesem Artikel den Anstoß von Eric Clark aufgreifen und entlang von drei Leitfragen Anregungen bieten: „[H]ow might urban governance contribute to making rent gap theory not true?" (Clark 2014). Dazu werden wir erstens bezogen auf den deutschen Kontext rekonstruieren, welche rechtlichen, stadtplanerischen und ökonomischen Voraussetzungen erfüllt sein müssen, dass Immobilieneigentümer_innen Mieten erhöhen und somit Ertragslücken schließen können, so dass in der Folge einkommensschwache Haushalte verdrängt werden. Es geht also darum theoretisch-deduktiv herzuleiten, wie sich in deutschen Städten das Entstehen und Schließen einer rent gap und damit Verdrängungsprozesse verhindern lassen (Abschnitt 2).

Zweitens werden wir empirisch am Beispiel des Frankfurter Gallus mittels kartographischer Darstellungen[1] aufzeigen, inwiefern und in welchem Ausmaß die Gültigkeitsbedingungen der Rent-Gap-Theorie im Zuge der Neoliberalisierung von Wohnungs- und Stadtplanungspolitiken in den letzten Jahrzehnten politisch hergestellt und die Ertragslückentheorie damit wahrgemacht worden ist (Abschnitt 3). Beim Gallus handelt es sich um ein klassisches, innerstädtisches Arbeiterviertel, welches trotz seiner zentralen Lage bis circa 2008 vergleichsweise niedrige Bestands- und Angebotsmieten sowie eine einkommensschwache Sozialstruktur aufgewiesen hat. Lange Zeit konnten Arbeiterhaushalte sowie Menschen mit niedrigem Einkommen, prekärem Aufenthaltsstatus und Migrationserfahrung hier bezahlbaren Wohnraum finden. Etwa seit 2010 lässt sich jedoch ein Prozess der NeubauGentrifizierung beobachten: Hochpreisiger Wohnungsbau auf dem Gelände des ehemaligen Güterbahnhofs (6.0oo Wohneinheiten) und auf verschiedenen weiteren Industriebrachen (2.00o Wohneinheiten) führt dazu, dass Investoren ihre stadtteilbezogenen Ertragserwartungen im Gallus an das hohe Niveau der umliegenden, bereits weitgehend gentrifizierten Viertel anpassen (Schipper/Wiegand 2015). Dementsprechend sind beispielsweise die Bodenpreise - als kapitalisierte Grundrente ein zentraler Indikator für zukünftige Renditeerwartungen (Schipper 2013b) - im Gallus zwischen 2014 und 2016 in nur zwei Jahren um bis zu 6o Prozent und damit deutlich überproportional gestiegen (Tab. 1).

Auch wenn der Gentrifizierungsdruck, der auf dem Viertel mittlerweile lastet, eindeutig belegt ist (Schipper/Wiegand 2015), bleibt die Frage offen, inwiefern die Bedingungen der Ertragslückentheorie in der Art erfüllt sind, dass die in jüngerer Zeit entstandene rent gap (ebd.: 1off.) auch tatsächlich zu umfassenden Verdrängungsprozessen führt. Im Gallus hat diese Frage eine besondere Brisanz, da es dort - wie noch detailliert aufgezeigt wird - vor 1989 selbst bei hohem Verwertungsdruck (bedingt durch die attraktive Lage innerhalb einer wachsenden Stadt) keine nennenswerte Gentrifizierung hätte geben können. Grund ist, dass sich damals knapp 6o Prozent der Wohnungen 
Tab. 1 Bodenpreissteigerungen an ausgewählten Frankfurter Standorten zwischen 2014 und 2016 (Quelle: Gutachterausschuss für Immobilienwerte der Stadt Frankfurt, eigene Berechnungen)

\begin{tabular}{|c|c|c|}
\hline Stadtteil & Standort & $\begin{array}{l}\text { Bodenpreissteigerung } \\
\text { 2014-2016 in Prozent }\end{array}$ \\
\hline Ostend & Sonnemannstraße & $65,65 \%$ \\
\hline Gallus & Koblenzerstraße & $60,00 \%$ \\
\hline Gutleutviertel & Mannheimer Straße & $55,30 \%$ \\
\hline Gallus & Frankenallee & $51,84 \%$ \\
\hline Innenstadt & Allerheiligenstraße & $50,59 \%$ \\
\hline Bornheim & Bornheimer Landwehr & $46,67 \%$ \\
\hline Sachsenhausen & Dreieichstraße & $46,67 \%$ \\
\hline Bockenheim & Jordanstraße & $40,51 \%$ \\
\hline Gallus & Philipp-Fleck-Straße & $39,37 \%$ \\
\hline Nordend & Nordendstraße & $38,04 \%$ \\
\hline Westend & Kettenhofweg & $36,93 \%$ \\
\hline Dornbusch & Fritz-Reuter-Straße & $35,88 \%$ \\
\hline Rödelheim & Schmittenerstraße & $35,28 \%$ \\
\hline Kalbach-Riedberg & Kalbacher Hauptstraße & $27,76 \%$ \\
\hline Heddernheim & Hammarskjöldring & $25,72 \%$ \\
\hline Eckenheim & Hochschildstraße & $25,10 \%$ \\
\hline Griesheim & Ahornstraße & $24,71 \%$ \\
\hline Niederrad & Adolf-Miersch-Straße & $24,59 \%$ \\
\hline Zeilsheim & Dessauer Weg & $19,06 \%$ \\
\hline Fechenheim & Schießhüttenstraße & $18,32 \%$ \\
\hline Schwanheim & Schwarzbachstraße & $18,32 \%$ \\
\hline Bonames & Ben-Gurion-Ring & $14,72 \%$ \\
\hline Unterliederbach & Euckenstraße & $14,72 \%$ \\
\hline Griesheim & Espenstraße & $13,88 \%$ \\
\hline Oberrad & Buchrainstraße & $13,88 \%$ \\
\hline Praunheim & Im Weimel & $13,12 \%$ \\
\hline Nied & Mainzer Landstraße & $12,67 \%$ \\
\hline Höchst & Hostatostraße & $7,59 \%$ \\
\hline Seckbach & Hofhausstraße & $7,23 \%$ \\
\hline
\end{tabular}

im Eigentum von nicht-gewinnorientierten beziehungsweise gemeinnützigen Akteuren befunden haben, deren Mieten preisgebunden waren. Da ein Großteil der Eigentümer_innen eine entstehende Ertragslücke aufgrund der Preisbindungen nicht über Mietsteigerungen hätten schließen können, war die rent gap im Gallus folglich „not true“ (Clark 2014). Im Kontext der tiefgreifenden Neoliberalisierung von Wohnungspolitiken in Deutschland wurde die Wohnungsgemeinnützigkeit allerdings 1989 auf Bundesebene abgeschafft und wurden die Preisbindungen damit aufgehoben. Vor dem Hintergrund eines sich parallel vollziehenden lokalpolitischen Wandels zur unternehmerischen Stadt in Frankfurt (Schipper 2013a) und eines Rückzuges aus dem sozialen Wohnungsbau wird daher analysiert, wie weitreichend im Gallus die Gültigkeitsbedingungen der Rent-Gap-Theorie durch Prozesse der Kommodifizierung, Privatisierung und Finanzialisierung politisch hergestellt worden sind, so dass sich der in den letzten Jahren entstandene Verwertungsdruck nun in Verdrängungsprozesse übersetzen kann.

Auf dieser Grundlage werden wir dann drittens erörtern, wie die stadtund wohnungspolitischen Rahmenbedingungen gestaltet sein müssten, damit die Rent-Gap-Theorie zukünftig keine Gültigkeit mehr beanspruchen kann und einkommensschwache Haushalte auch in Vierteln unter hohem 
Gentrifizierungsdruck effektiv vor Verdrängung geschützt sind (Abschnitt 4). Deutlich wird dabei, dass Gentrifizierung kein Naturgesetz darstellt, sondern ein zutiefst politischer Prozess ist, der sich durch eine Reform des Mietrechts, eine sozialgerechte Stadtplanung und eine Stärkung nicht-profitorientierter Wohnungsunternehmen steuern und auch effektiv verhindern lässt. Ebenso wird sichtbar, dass die marxistische Stadt- und Gentrifizierungsforschung nicht deterministisch beziehungsweise ökonomistisch argumentiert, sondern streng polit-ökonomisch, indem sie auf die gesellschaftliche Gewordenheit von Machtverhältnissen und deren mögliche Überwindung verweist (Slater 2017: 8f.).

\section{Die Rent-Gap-Theorie der Gentrifizierung und ihre Gültigkeitsbedingungen}

Gemäß der Bestimmung von Davidson und Lees (2005: 1187) kann immer dann von einer Gentrifizierung eines Stadtviertels gesprochen werden, wenn die Reinvestition von Kapital, der Zuzug einkommensstarker Gruppen sowie Veränderungen der urbanen Landschaft zu einer Verdrängung einkommensschwacher Schichten führen. In Anlehnung an die Typologien von Marcuse (1986) und Holm (2010: 6off.) können vier verschiedene Formen von Verdrängungsprozessen unterschieden werden.

Erstens findet eine direkte Verdrängung statt, wenn steigende Wohnkosten, die Umwandlung von Miet- in Eigentumswohnungen oder der physische Abriss von günstigem Wohnraum dazu führen, dass einkommensschwache Schichten ihre Wohnungen aufgeben und den Stadtteil verlassen müssen. Zweitens kann von indirekter Verdrängung gesprochen werden, wenn für einen Haushalt die eigenen Wohnkosten zwar bezahlbar bleiben, die Aufwertung und sozialräumliche Homogenisierung der Nachbarschaft aber zu einem Verlust sozialer Beziehungen, einer Veränderung der Gewerbestruktur sowie Entfremdungstendenzen führen, die letztlich in einer Wegzugsentscheidung münden (Atkinson 2015). Drittens beschreibt der Begriff der Verdrängung aus dem Lebensstandard eine Form der Gentrifizierung, in der Haushalte trotz steigender Wohnkosten in ihren Wohnungen verbleiben - sei es, weil sie sich eng mit dem Stadtteil verbunden fühlen, lokalräumliche existentielle soziale Netzwerke nicht aufgeben wollen oder weil sie auch im Rest der Stadt keine günstigere Wohnung finden können (Blasius 2004: 23). In diesem Fall kommt es also nicht zu einem Wegzug aus dem Stadtteil,jedoch zu einer Verschlechterung des Lebensstandards, da ein höherer Anteil des verfügbaren Haushaltseinkommens für die Miete ausgegeben wird, Zimmer an Schlafgänger_innen oder Airbnb-Touristen_innen untervermietet werden müssen oder weniger Wohnraum pro Person zur Verfügung steht. Viertens lässt sich von exkludierender Verdrängung sprechen, wenn in einem ehemaligen Arbeiterviertel mit traditionell relativ günstigen Mieten Schließungsprozesse stattfinden, die einkommensschwachen Haushalten den Zuzug verwehren.

\subsection{Die Rent-Gap-Theorie der Gentrifizierung}

Die Ursachen solcher Verdrängungsprozesse auf kleinräumiger Ebene politökonomisch erklären zu wollen, ist Anspruch der Rent-Gap-Theorie. Sie wurde 
ab Ende der 1970er Jahre von Neil Smith $(1979 ; 1996)$ und anderen Stadtforscher_innen (Clark 1995, Lees/Slater/Wyly 2008, Slater 2017) auf Basis empirischer Studien entwickelt, die in marktliberalen und meist angelsächsischen Kontexten mit schwachen Mieterrechten angesiedelt waren. Da die Rent-Gap-Theorie auf die Strukturen kapitalistischer Bodenmärkte sowie auf die Investitionsstrategien von Akteuren am Immobilienmarkt fokussiert, wird sie häufig als Gegenpol zu nachfrageorientierten Theorien der Gentrifizierung gelesen, die die räumlichen Präferenzen, Konsumentscheidungen und sich wandelnden Lebensstile von Mittelschichthaushalten ins Zentrum ihrer Erklärungsmodelle rücken (Frank 2017, Ley 1996). In Kontrast dazu begreift die Ertragslückentheorie Gentrifizierung als ein „back to the city movement by capital, not people" (Smith 1979), bei der die Bedürfnisse der Bewohner_innen mit den Profitinteressen von Investoren in Konflikt geraten (Slater 2017: 19).

Mittlerweile hat sich jedoch innerhalb der Gentrifizierungsforschung die Ansicht durchgesetzt, dass nachfrage- und angebotsorientierte Ansätze nicht zwingend als Gegensätze interpretiert werden müssen (vgl. Lees/Slater/ Wyly2008, Helbrecht 1996).Vielmehrkönnen siesich auch produktivergänzen, etwa wenn steigende Ertragserwartungen von Investoren mit veränderten Nachfragemustern und Lebensstilen von Haushalten der Mittel- und Oberschichten korrespondieren. Investoren bilden ihre Ertragserwartungen also nicht unabhängig von gesellschaftlichen Transformationsprozessen und Verschiebungen auf Seiten der potenziellen Nachfrager_innen. Die RentGap-Theorie hier in den Vordergrund zu rücken, resultiert somit nicht aus der Annahme, sie besäße eine umfassende Erklärungskraft. Der Grund liegt vielmehr darin, dass Inwertsetzungsstrategien der Angebotsseite leichter mittels staatlicher Regularien im Interesse einkommensschwacher Haushalte eingeschränkt und Verdrängungsprozesse damit effektiv unterbunden werden können. Alternative Strategien zur Beeinflussung der Nachfrage nach innerstädtischem Wohnen müssten letztlich auf die systematische Abwertung und Stigmatisierung von Stadtvierteln abzielen, was auch den Interessen einkommensschwacher Haushalte zuwiderliefe.

Konzeptionell knüpft die Rent-Gap-Theorie an den marxschen Begriff der Grundrente an, womit in Tradition der Kritik der politischen Ökonomie die Geldsumme benannt wird, „die der Grundeigentümer jährlich aus der Verpachtung [oder Vermietung, d. Verf.] eines Stücks des Erdballs bezieht“ (Marx 1988: 636). Marx ging dabei davon aus, dass sich in der Konkurrenz verschiedener Kapitalien um die Nutzung des Bodens in der Regel dasjenige Kapital durchsetzen wird, welches in der Lage ist, die höchste Grundrente in Form von Miete oder Pacht zu zahlen. Die innovative Leistung von Neil Smith besteht nun darin, zur Erklärung von Gentrifizierungsprozessen zwischen der gegenwärtigen und der potenziellen Grundrente zu unterscheiden. Während sich die gegenwärtige Grundrente - ganz im Sinne des Grundrentenbegriffs bei Marx - aus den gegenwärtig tatsächlich geleisteten Mietzahlungen speist, ist die potenzielle Grundrente bestimmt durch die antizipierte profitabelste Verwertung eines Grundstückes, die sich ergäbe, wenn sich zukünftig die gewinnträchtigste Nutzung durchsetzen würde. Die potenzielle Grundrente beruht somit auf den spekulativen Ertragserwartungen von Investoren. Gemäß der Rent-Gap-Theorie wird ein Stadtviertel für Immobilieninvestoren nun insbesondere dann für Aufwertungsstrategien interessant, wenn die 
Differenz zwischen gegenwärtiger und maximal möglicher Verwertung steigt (Clark 1995, Slater 2017, Smith 1996). Nur wenn die Ertragslücke groß genug geworden ist, lohnt sich ökonomisch das durchaus riskante, weil letztlich spekulative Geschäft, Wohnungsbestände aufzukaufen, gegebenenfalls zu modernisieren und am Ende teurer weiterzuvermarkten.

Ob sich ein derartiger ökonomischer Verdrängungsdruck jedoch auch tatsächlich - wie von der Rent-Gap-Theorie postuliert - in reale Verdrängungsprozesse übersetzt, ist keineswegs automatisch gesichert, sondern hängt von zahlreichen regulativen Kontextbedingungen ab. Selbige können sowohl die Entstehung als auch das Schließen einer Ertragslücke beeinflussen und dadurch die Gültigkeit der Rent-Gap-Theorie aufheben. Konsequent angewandt kann etwa eine mietrechtliche oder stadtplanerische Beschneidung des Mietzinses die spekulativen Ertragserwartungen und damit die potentielle Grundrente absenken und so die lagebedingte Entstehung einer rent gap blockieren. Relativ sinkende Boden- und Immobilienpreise wären die Folge. Zudem können regulative Rahmenbedingungen so gestaltet sein, dass ein Stadtviertel von nicht-gewinnorientierten Eigentümern dominiert wird, die wiederum kein Interesse an Mietsteigerungen haben. In diesem Fall bestünde weiterhin eine rent gap im Verhältnis zu den Ertragserwartungen gewinnorientierter Akteure, die sich empirisch etwa in deren Zahlungsbereitschaft für benachbarte Immobilien widerspiegelt. Eine nichtgewinnorientierte Eigentümerstruktur würde aber verhindern, dass sich in den entsprechenden Beständen rent gaps über steigende Mieten schließen.

\section{2. ...und ihre Gültigkeitsbedingungen im deutschen Kontext}

Wahr in dem Sinne, dass wachsende Ertragslücken zwischen gegenwärtiger und potenzieller Grundrente entstehen können und zu Verdrängung führen, wird die Rent-Gap-Theorie nur dann, wenn (1) die profitabelste Bewirtschaftung von Wohnimmobilien nicht durch staatliche Regularien beschränkt wird, (2) eine an Investoreninteressen ausgerichtete Stadtplanung insbesondere bei Neubauprojekten die gewinnbringendste Nutzung des Bodens ermöglicht und (3) eine Eigentümerstruktur vorherrscht, die Mieterhöhungsspielräume durchsetzen will und gegebenenfalls notwendige Modernisierungsmaßnahmen auch finanzieren kann.

\section{Mietrecht und Bestandswohnungen}

Eine staatliche Regulation des Wohnungsmarktes kann das Entstehen und Schließen von Ertragslücken und damit Verdrängungsprozesse verhindern, indem Mieterhöhungsspielräume beschränkt werden. Das eindrücklichste Beispiel hierfür ist die Wohnungszwangswirtschaft, wie sie etwa in Deutschland nach dem Ersten Weltkrieg etabliert wurde. Gemäß dem Reichsmietengesetz von 1922 waren die Mieten von Altbauwohnungen auf dem Niveau der sogenannten Friedensmiete vom 1. Juli 1914 eingefroren und konnten lediglich im Rahmen von Mehrbelastungen durch steigende Bewirtschaftungskosten angepasst werden. Schrittweise abgeschafft wurde die staatliche Fixierung der Bestandsmieten in der Bundesrepublik erst mit der Verabschiedung des „Gesetzes über den Abbau der Wohnungszwangswirtschaft“ im Juni 1960 (Brede/Kohaupt/Kujath 1975: 64ff.). Die historischen 
Erfahrungen mit derartigen Preisbeschränkungen im Mietwohnungssektor zeigen, dass selbige staatlicherseits relativ leicht durchgesetzt werden können, da sich das in Immobilien investierte Kapital den Vorgaben nur schwer entziehen kann. Eine Mietpreisbegrenzung für Bestandswohnungen stellt eine „der effektivsten Formen staatlicher Intervention zugunsten der Reproduktionsbedinungen der Bevölkerung“ (Brede/Kohaupt/Kujath 1975: 52f.) dar, weil in Immobilien gebundenes fixes Kapital „relativ unelastisch“ (ebd.: 58) gegenüber staatlichen Eingriffen und einer zwangsweisen Absenkung des Mietzinses ist. Solange die Mieten inflationsbereinigt nicht real sinken, bleibt darüber hinaus auch das investierte Kapital erhalten und die Bausubstanz gesichert (ebd.: 73).

Die moderatere und verbreitetere Form der Mietenregulation besteht hingegen darin, nicht die absolute Miete zu fixieren, sondern lediglich die Erhöhungsspielräume zu begrenzen. Gedacht als Kompromiss zwischen den Verwertungsinteressen der Vermieter_innen einerseits und dem Schutzbedürfnis der Mieter_innen andererseits, können etwa Mieten in Deutschland nur um maximal 2o Prozent in drei Jahren angehoben werden und dürfen die ortsübliche Vergleichsmiete nicht überschreiten. Seit März 2013 gilt zudem auf angespannten Wohnungsmärkten eine verschärfte Kappungsgrenze von maximal 15 Prozent. Bezogen auf mögliche Gentrifizierungsgebiete bedeutet dies, dass direkte Verdrängungsprozesse durch reguläre Mieterhöhungen zwar gesetzlich nicht verhindert werden. Jedoch wird das Schließen einer rent gap zeitlich abgebremst, da drastische Mieterhöhungen per Gesetz nicht zulässig sind (Blasius 2004: 34f.). Dementsprechend kommen etwa Jürgen Friedrichs und Jörg Blasius (2016) in ihrer Langzeitstudie zu Gentrifizierung in Köln zu dem Ergebnis, dass aufgrund „der relativ mieterfreundlichen Gesetze“ (ebd.: 24) Phasen der Verdrängung „in Deutschland wesentlich länger als in anderen Ländern dauern“ (ebd.: 21), in denen „nahezu ausschließlich der Markt entscheidet, zu welchem Preis eine Wohnung vermietet oder verkauft werden kann“ (ebd.: 24).

Allerdings existieren mehrere Ausnahmeregelungen, die diesen Befund abschwächen. Beispielsweise können Eigentümer_innen laut Mietrecht 11 Prozent der Kosten für Modernisierungsmaßnahmen pro Jahr auf die Mieter_innen umlegen. Insbesondere in Aufwertungsgebieten kann dies ein beliebtes Instrument sein, um Mieten drastisch zu erhöhen und unerwünschte Altmieter_innen zu verdrängen. Hier reicht häufig schon eine bloße Ankündigung von Modernisierungsmaßnahmen, um Mieter_innen zum Auszug zu bewegen (Blasius 2004: 36). Zudem können günstige Mietverhältnisse beendet werden, in dem (real oder fingiert) Eigenbedarf angemeldet wird. Als Kündigungsgrund gilt weiterhin, wenn sich die Eigentümer_innen in ihrer angemessenen wirtschaftlichen Verwertung des Grundstücks gehindert sehen. Ebenso können Mietwohnungen aufgekauft und dann - je nach Bundesland nach einer Frist von drei bis zehn Jahren in Eigentumswohnungen umgewandelt werden.

Zusammenfassend lässt sich festhalten, dass reguläre Mieterhöhungen durch das deutsche Mietrecht begrenzt, aber nicht ausgeschlossen sind. Dadurch wird das Schließen von rent gaps zwar insgesamt verlangsamt, aber nicht dauerhaft blockiert. Direkte Verdrängung aus einer Bestandswohnung qua Mieterhöhung tritt in Nachbarschaften unter Gentrifizierungsdruck 
somit weniger flächendeckend auf (Friedrichs/Blasius 2016), sondern ist - vor allem bedingt durch die genannten Ausnahmeregelungen - ein punktuell voranschreitendes Phänomen. Zu vermuten ist außerdem, dass Gentrifizierung in deutschen Großstädten häufig durch die betroffenen Haushalte als sukzessive Verdrängung aus dem Lebensstandard erlebt wird (Blasius 2004), weil trotz allem die Bestandsmieten schrittweise steigen. Da ferner die Angebotsmieten - trotz neu eingeführter Mietpreisbremse - praktisch nicht reguliert sind, kann das bestehende Mietrecht exkludierende Verdrängungsprozesse und das Entstehen von rent gaps nicht verhindern. Durch den Verlust preisgünstigen Wohnraums bei Neuvermietung kommt es in Gentrifizierungsgebieten vielmehr zu einer schleichenden Veränderung der Sozialstruktur (Mösgen/Schipper 2017: 13of.).

Um Gentrifizierung qua Mietrecht zu verhindern, müsste unter anderem auf Bundesebene bei Neuvermietungen eine wirksame Mietpreisbremse etabliert, für Bestandsverträge die Kappungsgrenze weiter abgesenkt, die Bemessungsgrundlage von Mietspiegeln auf alle Mietverträge ausgedehnt, die Modernisierungsumlage von derzeit 11 Prozent gestrichen sowie auf Landesebene ein Umwandlungsverbot von Miet- in Eigentumswohnungen eingeführt werden. Eine derartige Reform des Mietrechts würde bewirken, dass die Gültigkeitsbedingungen der Rent-Gap-Theorie nicht mehr erfüllt wären, da sich Mieten nicht nennenswert erhöhen und sich die profitabelste Nutzung eines Wohngebäudes nicht unmittelbar durchsetzen ließen.

\section{Stadtplanung und Neubau-Gentrifizierung}

Ohne staatliche Interventionen tendieren Immobilieninvestoren dazu, beim Neubau von Wohnungen ausschließlich das gehobene Segment teurer Mietund Eigentumswohnungen zu bedienen, weil hier diehöchsten Gewinnspannen zu erzielen sind. Unter den Bedingungen eines freien Bodenmarktes setzt sich daher in Konkurrenz um Bauland stets die profitabelste Nutzung durch, weil mit hohen Mieten und Wohnungspreisen die höchsten Grundrenten realisiert und damit auch die höchsten Bodenpreise gezahlt werden können (Harvey 2006: 330ff.). Nicht-gewinnorientierten Bauträgern, wie etwa Genossenschaften und anderen kollektiven Eigentumsmodellen, wird vermittelt über den Bodenpreismechanismus die Schaffung preisgünstigeren Wohnraums verwehrt (Balmer/Bernet 2017). In Konsequenz kommt es in Neubaugebieten zu einer exkludierenden Verdrängung, weil weniger privilegierte Haushalte systematisch vom Zuzug ausgeschlossen werden. Zudem kann es in der unmittelbaren Nachbarschaft unter Umständen zu einer Neubau-Gentrifizierung kommen, insofern der gehobene Wohnraum auch Aufwertungstendenzen in der Umgebung initiiert (Davidson/Lees 2005, Schipper/Wiegand 2015).

Ob sich im Neubau allerdings die profitabelste Nutzung durchsetzen kann und somit rent gaps geschlossen werden, hängt wesentlich von den stadtplanerischen und wohnungspolitischen Rahmenvorgaben ab. Das oben beschriebene Phänomen exklusiver Neubauviertel tritt nur dann ein, wenn (a) öffentliche Liegenschaften zum Höchstgebotsverfahren ohne sozialpolitische Auflagen privatisiert werden und wenn (b) auf Grundstücken in privatem Eigentum die profitabelste Nutzung stadtplanerisch nicht eingeschränkt wird. Zu beiden Gültigkeitsbedingungen existieren jedoch politische Alternativen. 
Zum einen müssen öffentliche Liegenschaften nicht in einem Höchstgebotsverfahren an den zahlungskräftigsten Investor veräußert werden, um etwa einen möglichst hohen Spekulationsgewinn in die Stadtkasse zu spülen. Kommunen können als Eigentümerinnen stattdessen ihre Grundstücke auch mit klaren sozialpolitischen Auflagen entweder in Erbpacht oder nach Konzeptqualität an Bauträger vergeben, die sich verpflichten, einen gewissen Anteil preisgebundener oder preisgünstiger Wohnungen zu realisieren. Liegenschaften, die sich bereits in kommunalem Besitz befinden oder strategisch angekauft werden, können also unter Umgehung des Bodenpreismechanismus Akteuren überlassen werden, die den vorab definierten sozialpolitischen Qualitätskriterien am weitestgehenden genügen und die Schaffung preisgünstigen Wohnraums garantieren.

Zum anderen können Kommunen planungsrechtlich über die Aufstellung von Bebauungsplänen die Nutzungsart sowie die maximal zulässige Bebauungsdichte von Grundstücken bestimmen und dadurch die potenziell erzielbare Grundrente beeinflussen. Auf Grundstücken in privatem Eigentum, auf denen neues Baurecht geschaffen wird, erlaubt zudem das Planungsrecht, den per Federstrich erzeugten Bodenwertzuwachs, der beispielsweise bei der Umwidmung von Gewerbeflächen in Wohngebiete entsteht, zu einem gewissen Grad zugunsten öffentlicher Interessen abzuschöpfen. Gemeinden können demnach über städtebauliche Verträge nach § 11 BauGB Investoren an den Erschließungskosten und der Finanzierung sozialer Infrastrukturen beteiligen sowie zur Schaffung geförderten und preisgebundenen Wohnraums verpflichten (Helbrecht 2017).

Mit den genannten Instrumenten der Liegenschafts- und Planungspolitik können Kommunen also potenzielle Grundrenten schmälern und damit Ertragslücken verringern. Dadurch lassen sich sowohl exkludierende Verdrängungsprozesse als auch die Neubau-Gentrifizierung der umliegenden Nachbarschaften verhindern oder zumindest abmildern.

\section{Eigentümerstruktur}

Schließlich kann die Rent-Gap-Theorie nur Gültigkeit beanspruchen, wenn die Eigentümerstruktur in einem Stadtviertel von gewinnorientierten Akteuren dominiert wird, die sowohl ein Interesse an der Schließung von Ertragslücken haben, als auch über das notwendige Kapital für gegebenenfalls notwendige Aufwertungsmaßnahmen verfügen. Wie die folgenden vier Kategorien an möglichen Eigentümerstrukturen zeigen, ist die Dominanz einer renditeorientierten Wohnungswirtschaft, die Gentrifizierungsprozesse anzutreiben vermag, auch in kapitalistischen Gesellschaften keineswegs selbstverständlich und alternativlos.

- Als profitorientierte, institutionelle Investoren sind alle privatwirtschaftlichen Wohnungsunternehmen, Kreditinstitute, Versicherungen, börsennotierte Immobilienunternehmen, Immobilienfonds sowie weitere Kapitalgesellschaften zu bezeichnen, die systematisch auf Gewinnmaximierung ausgerichtet sind. Aufgrund der klaren Shareholder-Value-Orientierung sind diese Akteure strukturell auf die Optimierung ihrer Renditen ausgerichtet, weshalb sie aktiv bestrebt sind, rent gaps zu identifizieren und systematisch durch Erhöhungen der Mieten zu schließen (Fields/Uffer 2016, Unger 2016). Da sie zudem 
direkt an Kapitalmärkte angebunden sind, verfügen diese Investoren in der Regel auch über die notwendigen finanziellen Mittel, um etwa umfangreiche Modernisierungs- und Bauvorhaben zur Aufwertung der Gebäudesubstanz realisieren zu können.

- Private Kleineigentümer_innen sind als Amateurvermieter_innen dadurch charakterisiert, dass sie nur jeweils einen überschaubaren Immobilienbesitz von einzelnen Wohnungen oder wenigen Mietshäusern aufweisen. Ob sie in Gentrifizierungsgebieten von den durch eine rent gap entstandenen Mieterhöhungs- und Aufwertungsspielräumen Gebrauch machen, kann im Gegensatz zur Geschäftspraxis institutioneller Investoren auf theoretischer Ebene nicht eindeutig beantwortet werden. Da sie keiner strukturell-determinierenden Logik unterliegen, bleibt es eine individuelle, subjektive Entscheidung, inwiefern Aufwertungspotenziale für Mieterhöhungen und Modernisierungsmaßnahmen auch tatsächlich ausgeschöpft werden. Anzunehmen ist sicherlich, dass manche Kleineigentümer_innen sich eröffnende Potenziale, Mieten ans Marktniveau anzupassen, konsequent ausnutzen oder sogar weitergehende legale oder illegale Strategien zur Mieterverdrängung anwenden, um von Gentrifizierungsprozessen finanziell zu profitieren. Anzunehmen ist aber ebenso, dass auf der anderen Seite eines breiten Spektrums von Handlungsmöglichkeiten Kleineigentümer_innen agieren, die aus sozialem Gewissen, persönlicher Verbundenheit mit ihren Mieter_innen oder einfach aus Bequemlichkeit den Mietzins über einen langen Zeitraum nicht oder kaum erhöhen. Inwiefern sich eine rent gap in Gebieten, deren Eigentümerstruktur von privaten Amateurvermieter_innen dominiert wird, schließt, lässt sich daher nicht deduktiv-theoretisch beantworten, sondern muss jeweils im Einzelfall empirisch untersucht werden.

- Öffentliche Wohnungsunternehmen befinden sich in kommunalem oder staatlichem Eigentum. Für sich genommen sagen die Eigentumsverhältnisse allerdings wenig über die jeweilige Geschäftspraxis aus. Öffentliche Unternehmen können beispielsweise in Analogie zu privatwirtschaftlichen Investoren auf Gewinnorientierung ausgerichtet sein, um öffentliche Haushalte zu entlasten oder um gehobenen Wohnraum für einkommensstarke Schichten zu realisieren. Andererseits können sie aber auch zu einem sozialen Versorgungsauftrag verpflichtet und gezielt angewiesen werden, bezahlbaren Wohnraum für diejenigen zu sichern, die sich nicht selbst am Markt versorgen können. Ob öffentliche Wohnungsunternehmen die Entstehung einer rent gap ausnutzen, um Mieten zu erhöhen und den eigenen Bestand aufzuwerten, eine solche Entwicklung womöglich sogar aktiv herbeiführen oder umgekehrt Gentrifizierungsprozessen entgegenwirken, ist daher eine politisch zu treffende Entscheidung. Sie ist abhängig von den jeweiligen Zielsetzungen der Exekutive und den parlamentarischen Mehrheitsverhältnissen.

- Die Prinzipien einer gemeinnützigen und nicht-gewinnorientierten Wohnungswirtschaft sind im 19. Jahrhundert zuerst als freiwillige Selbstverpflichtung einzelner philanthropischer Wohnungsunternehmen und Baugenossenschaften der Arbeiterbewegung entstanden. Um eine profitförmige Verwertung von Wohnraum zu verhindern, haben sich 
gemeinnützige Wohnungsunternehmen bei der Mietpreisbildung am Grundsatz der Kostendeckung statt der Gewinnmaximierung ausgerichtet, die Verzinsung des Eigenkapitals auf maximal vier Prozent begrenzt, das Vermögen verbindlich in die fortwährende Weiterentwicklung der Wohnungsbestände investiert und als zu versorgende Zielgruppen untere und mittlere Einkommensschichten definiert. Eine gesetzlich verbindliche Kodifizierung der gemeinnützigen Wohnungswirtschaft erfolgte mit der Gemeinnützigkeitsverordnung von 1930 und dem Wohnungsgemeinnützigkeitsgesetz (WGG) von 1940, welches in der Bundesrepublik Deutschland als zentrales Instrument der Wohnungspolitik bis 1990 fortbestand (Kuhnert/Leps 2017). Geprägt war die gemeinnützige Wohnungswirtschaft von einer Vielzahl an Unternehmensformen, wie etwa öffentlichen Wohnungsunternehmen, Genossenschaften, Stiftungen und privatwirtschaftlichen Werkswohnungen. Von den bis 1989 in der Bundesrepublik errichteten circa 19 Millionen Wohnungen wurden knapp 4,8 Millionen von gemeinnützigen Unternehmen fertiggestellt (Holm/Horlitz/Jensen 2015: 7). Damit verfügten gemeinnützige Unternehmen in Großstädten mit mehr als 100.00o Einwohner_innen im Durchschnitt über einen Marktanteil von 30 Prozent (Kuhnert/Leps 2017: 54). Dadurch war ein signifikanter Anteil der Mieterhaushalte vor potenziellen Verdrängungsprozessen geschützt. Im Zuge der Steuerreform von 1990 beschloss die damalige CDU/ FDP-Regierung unter Helmut Kohl allerdings, die Gemeinnützigkeit im Wohnungssektor abzuschaffen. In Folge gingen Millionen preisgebundener Wohnungen verloren, da viele ehemals gemeinnützige Unternehmen nach Abschaffung der gesetzlichen Preisbindungen privatisiert oder gewinnorientiert restrukturiert worden sind (Kuhnert/ Leps 2017, Unger 2016). Eine wichtige Ausnahme zu dieser Entwicklung bilden Genossenschaften und andere kollektive Eigentumsmodelle wie etwa das „Mietshäuser Syndikat“. Aufgrund des Identitätsprinzips, nach dem die Mitglieder kollektiv Eigentümer_innen und zugleich auch Mieter_innen sind, besteht strukturell kein Anreiz für Mieterhöhungen (Balmer/Bernet 2017). Derartige Akteure am Wohnungsmarkt sind daher auch nach Abschaffung der gesetzlichen Preisbindungen aufgrund der kollektiven Selbstverwaltungsstrukturen weiterhin nicht-gewinnorientiert ausgerichtet und weisen demzufolge bis heute relativ günstige Mieten auf. Auch in Gentrifizierungsgebieten haben sie kein Interesse an einer Schließung von wachsenden Ertragslücken. Ebenso können Stiftungen und kirchliche Träger nach den Gemeinnützigkeitsprinzipien agieren, aber hier ist - in Analogie zu öffentlichen Wohnungsunternehmen - jeweils empirisch zu prüfen, unter welchen Maximen das Immobilienvermögen bewirtschaftet wird.

Zusammenfassend kann die Rent-Gap-Theorie also nur Gültigkeit in dem Sinne beanspruchen, dass wachsende Ertragslücken potenziell auch zu Mietsteigerungen und Verdrängung führen, wenn wesentliche Bedingungen im Mietrecht, bei stadtplanerischen Entscheidungen und bezüglich der Eigentümerstruktur erfüllt sind. Anders formuliert, lösen ansteigende Differenzen zwischen gegenwärtigen und potenziellen Grundrenten umso 
gravierendere Gentrifizierungsprozesse aus, je schwächer die Verwertung von Wohnimmobilien mietrechtlich reguliert ist, je investorenfreundlicher die lokale Stadtplanung agiert und je stärker die Eigentümerstruktur eines Viertels von profitorientierten Akteuren dominiert wird.

Wie diese Gültigkeitsbedingungen der Rent-Gap-Theorie im Zuge tiefgreifender Neoliberalisierungsprozesse politisch hergestellt worden sind und welche Konsequenzen das hat, werden wir im Folgenden am Beispiel des Frankfurter Gallus analysieren. Unsere These lautet, dass die Abschaffung der Wohnungsgemeinnützigkeit und der Wegfall der Preisbindungen sowie verschiedene weitere stadtplanerische Interventionen und lokalpolitische Entscheidungen seit Anfang der 1990er Jahre die Voraussetzungen dafür erzeugt haben, dass Eigentümer_innen gegenwärtig die jüngst entstandene rent gap über Mietsteigerungen schließen können und in Folge Verdrängungsprozesse einsetzen. Wir argumentieren also, dass es vor 1989 selbst bei hohem Gentrifizierungsdruck keine nennenswerte Verdrängung hätte geben können, sondern erst durch eine grundlegende Transformation der Eigentümerstrukturen im Gallus der Weg für die jüngste Welle der Gentrifizierung geebnet worden ist.

\section{Fallbeispiel: Gentrifizierung und Verdrängung im Frankfurter Gallus}

Das Frankfurter Gallus mit heute über 36.00o Einwohner_innen ist Ende des 19. Jahrhunderts im Zuge der Industrialisierung als klassisches Arbeiterviertel entstanden. In unmittelbarer Nähe zu zahlreichen Gewerbe-, Handwerksund Industriebetrieben, die sich ab den 1880er Jahren westlich des Hauptpersonen- und Hauptgüterbahnhofes angesiedelt hatten, errichteten damals vor allem gemeinnützige Wohnungsbaugesellschaften eine Vielzahl von einfachen Wohnungen für die in den Fabriken arbeitende Bevölkerung. Durch das Wohnungsgemeinnützigkeitsgesetz unterlagen die Mieten dieser Wohnungen einer Preisbindung im Sinne des Kostenmietenprinzips. Bei den Wohngebäuden im Besitz von privaten Kleineigentümer_innen führte zudem der Mangel an Grünflächen und sozialen Infrastrukturen sowie die hohe Luftverschmutzung und Lärmbelastung im Viertel zu einem relativ niedrigen Mietniveau bei gleichzeitig geringem Ausstattungsstandard (Sozialwissenschaftliche Arbeitsgruppe Mühlich \& Mühlich 1974).

Ab den 1970er Jahren setzten im Gallus zwei grundlegende Transformationsprozesse ein. Zum einen kam es zu einer rasanten Deindustrialisierung des Viertels. Durch den Rückgang des produzierenden Gewerbes gingen bis 1987 6o Prozent der Arbeitsplätze verloren und ein Großteil der ehemaligen Industrieflächen fiel brach (Schipper/Wiegand 2015: 12). Zum anderen begann sich die Bevölkerungsstruktur zu ändern. Während die besserverdienende Facharbeiterschaft sowie die Angestellten und Beamten tendenziell ins suburbane Umland abwanderten, stieg parallel dazu der Anteil der Arbeitsmigrant_innen auf knapp 42 Prozent der Wohnbevölkerung (ebd.: 14). Angesichts eines negativen, häufig rassistisch konnotierten Images wurde das Viertel trotz seiner innerstädtischen Lage von einkommensstärkeren Bevölkerungsgruppen als unattraktiv eingeschätzt (Ronneberger/Keil 1995: 327; Sozialwissenschaftliche Arbeitsgruppe Mühlich 
\& Mühlich 1974: 175ff.) - mit dem positiven Nebeneffekt, dass die Mietwohnungen auch für einkommensschwache Haushalte relativ bezahlbar blieben. Zwar setzte im Kontext der Global-City-Formierung Frankfurts ab Mitte der 1980er Jahre eine positive Neubewertung des Gallus als potenzieller Bürostandort für den expandierenden Finanzdistrikt ein, wodurch es zu einer kurzen Phase spekulativ steigender Bodenpreise kam. Frühe Bestrebungen zur Aufwertung und Gentrifizierung des Stadtteils scheiterten jedoch mit dem Platzen der Immobilienblase Anfang der 1990er Jahre (Schipper 2013b).

Erst im Anschluss an die globale Finanzkrise von 2008 ist das Gallus erneut unter erheblichen Gentrifizierungsdruck geraten (Schipper/Wiegand 2015). Selbiger offenbart sich gegenwärtig in überproportional steigenden Bodenpreisen (Tab. 1) und wachsenden Angebotsmieten. Vor allem der hochpreisige Wohnungsneubau im Europaviertel auf dem Areal des früheren Hauptgüterbahnhofes sowie auf anderen ehemaligen Industriebrachen im Viertel hat dazu geführt, dass sich die Ertragserwartungen von Immobilieninvestoren an das hohe Niveau der umliegenden Stadtteile angeglichen haben. Entstanden ist so eine rent gap zwischen den traditionell vergleichsweise niedrigen Bestandsmieten einerseits sowie der potenziellen Verwertung im Hochpreissegment andererseits - eine Ertragslücke, die nun Gentrifizierungsprozesse antreibt. Vor dem Hintergrund dieser Diagnose werden wir im Folgenden analysieren, inwiefern die in Abschnitt 2 benannten Gültigkeitsbedingungen für Gentrifizierungsprozesse im Gallus derart erfüllt sind, dass die jüngst entstandene rent gap tatsächlich auch in Verdrängungsprozesse mündet.

\subsection{Methodisches Vorgehen}

Ausgangspunkt der Untersuchung bildet eine unveröffentlichte Studie, die 1974 im Auftrag der Stadt Frankfurt von der Sozialwissenschaftlichen Arbeitsgruppe Mühlich \& Mühlich erstellt worden ist und die uns die Autor_innen freundlicherweise inklusive dem gesamten Datenmaterial zugänglich gemacht haben. Zweck der Untersuchung war damals, einen Beitrag zur Strukturplanung im Gallus zu leisten und die „Grundlagen für die Formulierung und Begründung von Entwicklungsalternativen“" (Sozialwissenschaftliche Arbeitsgruppe Mühlich \& Mühlich 1974: 94) zu erarbeiten. Ermöglicht haben uns die Studie sowie das dazugehörige Rohmaterial, die historische Entwicklung der Eigentümerstruktur sowie den ebenfalls dokumentierten Wandel der Sozialstruktur seit Beginn der Industrialisierung kleinräumig nachzuvollziehen. Besonders ergiebig war die Untersuchung für den Zweck unserer Fragestellung vor allem deshalb, weil die Autor_innen sowohl auf die amtliche Gebäude- und Wohnungszählung von 1968 zurückgreifen konnten als auch eine eigenständige Befragung (Stand 1973/74) aller elf damals im Gallus aktiven gemeinnützigen Wohnungsbaugesellschaften, Genossenschaften und Stiftungen durchgeführt haben (für eine Auflistung siehe Abb. 1). Detaillierten Aufschluss erhalten haben wir so über deren Gebäude- und Wohnungsbestand, die Bewirtschaftungspraxis, die Miethöhe, die Mieterstruktur und den Baustandard. Demnach bewirtschafteten die nicht-gewinnorientierten Wohnungsunternehmen im Gallus Mitte der 1970er Jahre mit 7.801 von 13.338 Wohneinheiten knapp 58,5 Prozent des 
gesamten Wohnungsbestandes (Sozialwissenschaftliche Arbeitsgruppe Mühlich \& Mühlich 1974: 166). Die restlichen gut 40 Prozent der Wohnungen waren im Besitz privater Kleineigentümer_innen. Bezüglich der Amateurvermieter_innen sind im Rahmen der Studie zwar keine Erhebungen zur Bewirtschaftungspraxis durchgeführt worden. Allerdings konnten Mühlich \& Mühlich auch hier das Mietniveau und den Baustandard der Wohnungen erfassen. Kartographisch dargestellt ist die Eigentumsstruktur der 1970er Jahre in Abbildung 1.

Aufbauend auf dieser Studie und kontrastierend zur damaligen Situation haben wir anschließend versucht, die heutige Eigentümerstruktur im Gallus zu erfassen. Dazu wurden erstens die 11 ehemals gemeinnützigen Wohnungsunternehmen, die bereits in den 1970er Jahren über Wohnungsbestände im Gallus verfügten, beziehungsweise deren rechtliche Nachfolgerinnen, erneut telefonisch kontaktiert und die damalige Befragung wiederholt, um Erkenntnisse über Veränderungen bezüglich deren Wohnungsbeständen (Zukäufe, Neubau, Veräußerungen) sowie ihrer gegenwärtigen Bewirtschaftungspraxis zu gewinnen. Insofern die ehemals gemeinnützigen Unternehmen Liegenschaften verkauft hatten, wurden ebenso die neuen Eigentümer kontaktiert. Bemerkenswerterweise haben uns sämtliche Akteure inklusive dem privaten, börsennotierten Großunternehmen Vonovia[2] (früher Deutsche Annington), welches im Gallus die ehemaligen Werkswohnungen der Gemeinnützigen Bundesbahn-Wohnungsgesellschaft übernommen hat, bereitwillig Auskunft erteilt. Lediglich die drei öffentlichen Wohnungsunternehmen ABG Frankfurt Holding, Nassauische Heimstätte und GWH Wohnungsbaugesellschaft haben jegliche Kooperation verweigert und keine Informationen zur Verfügung gestellt, sodass wir diesbezüglich auf andere, öffentlich zugängliche Quellen (Jährliche Geschäftsberichte, Pressemitteilungen, etc.) zurückgreifen mussten. In einem zweiten Schritt haben wir anschließend recherchiert, welche Immobilienentwickler auf den
Abb. 1 Die Eigentumsverhältnisse im Gallus Mitte der 1970er Jahre (alle Karten: Darstellung Elke Alban)

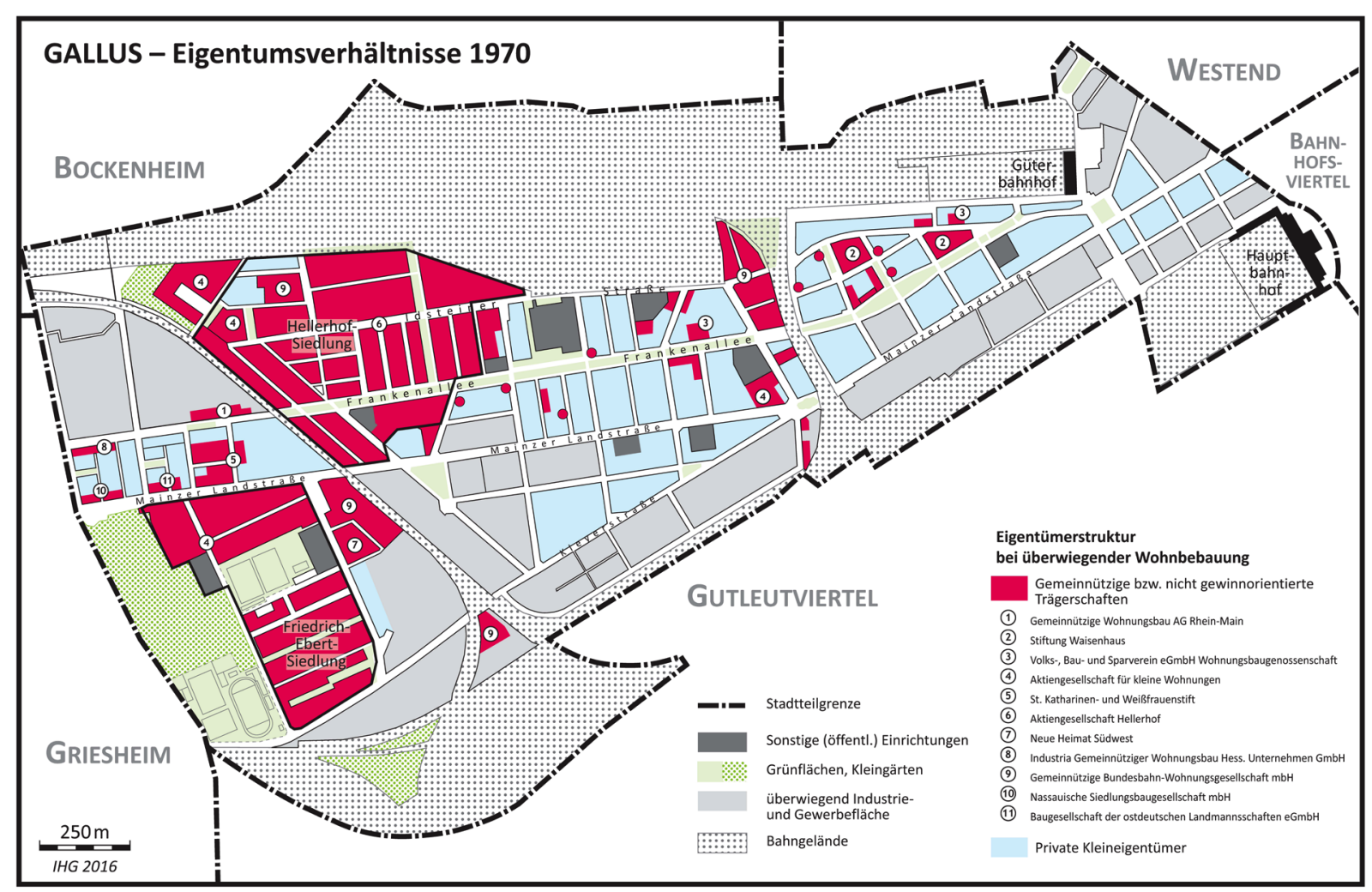


ehemaligen Industriebrachen und Gewerbeflächen sowie im Europaviertel Neubauprojekte realisiert haben. Eine Auswertung der Lokalpresse, die Webauftritte der entsprechenden Unternehmen sowie das Deutsche Architekturforum[3] waren hier besonders ergiebige Quellen. In unklaren Fällen hat zudem drittens eine Begehung vor Ort und die Auswertung der entsprechenden Bauschilder Aufschluss über die Eigentumsverhältnisse gegeben. Schließlich haben wir viertens über stichprobenartige Anfragen beim Katasteramt analysiert, inwiefern sich der Bestand an Wohnungen im Besitz privater Kleineigentümer_innen seit Mitte der 1970er Jahre verändert hat und ob sich dort beispielsweise institutionelle Investoren, öffentliche Unternehmen oder andere Akteure in nennenswertem Umfang eingekauft haben. Das Ergebnis unserer eigenen Untersuchung der gegenwärtigen Eigentümerstruktur im Gallus (Stand 2016) ist in Abbildung 2 dargestellt.

\subsection{Die Eigentümerstruktur im Gallus in den 1970er Jahren}

Mitte der 1970er Jahre befanden sich knapp 6o Prozent aller Wohnungen im Gallus im Eigentum von nicht-gewinnorientierten Akteuren, deren Mieten gemäß den Vorgaben des Wohnungsgemeinnützigkeitsgesetzes (WGG) einer Preisbindung unterlagen. Die restlichen gut 40 Prozent waren im Besitz privater Kleineigentümer_innen. Die räumliche Verteilung, an der sich bis zur Abschaffung der Wohnungsgemeinnützigkeit 1989 wenig verändert hat, ist in Abbildung 1 dargestellt. Angesichts dieser Eigentümerstruktur ist davon auszugehen, dass eine infrastrukturelle und stadtplanerische Aufwertung des Viertels unter damaligen Bedingungen kaum zu Verdrängung geführt hätte. Da die gemeinnützigen Wohnungsunternehmen gesetzlich auf die Kostenmiete

Abb. 2 Die Eigentumsverhältnisse im Gallus Stand 2016 verpflichtet waren, hätten sie potenzielle Mieterhöhungsspielräume nicht ausnutzen dürfen. Somit wäre ein Großteil der Bewohner_innen unmittelbar vor Verdrängungsdruck geschützt gewesen. Angesichts der dominierenden

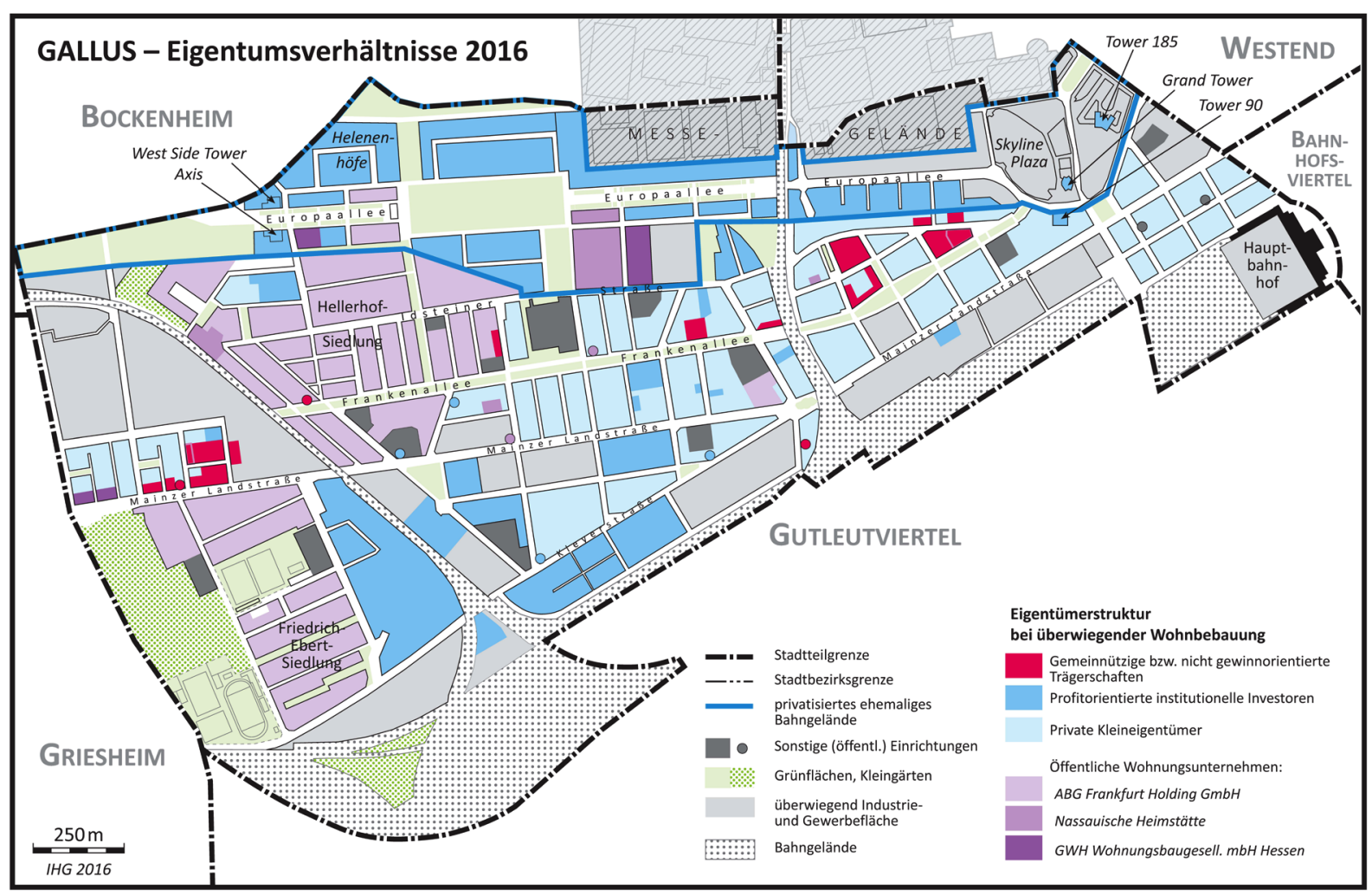


Stellung des nicht-gewinnorientierten Sektors und der daraus resultierenden relativ niedrigen ortsüblichen Vergleichsmiete hätten zudem auch die privaten Kleineigentümer_innen Schwierigkeiten gehabt, drastische Mieterhöhungen auszusprechen, da Mieter_innen stets die Alternative gehabt hätten, in die günstigeren Bestände des gemeinnützigen Sektors in unmittelbarer Nachbarschaft umzuziehen.

Insgesamt hat bis 1989 also die spezifische Eigentümerstruktur sichergestellt, dass Mieten nicht ans Marktniveau angepasst und folglich Ertragslücken nicht hätten geschlossen werden können. Das Gallus war daher unter damaligen Bedingungen nicht gentrifizierbar.

\subsection{Wie Prozesse der Neoliberalisierung die Rent-Gap-Theorie wahr gemacht haben}

Seit 1990 hat jedoch die Neoliberalisierung von Wohnungs- und Stadtplanungspolitiken zu einem tiefgreifenden Wandel der Eigentümerstruktur im Gallus geführt. Während der nicht-gewinnorientierte Sektor dramatisch geschrumpft ist, prägen nun neben privaten Kleineigentümer_innen vor allem institutionelle Investoren die Wohnraumversorgung im Stadtteil (Abb. 2). Konkret haben drei wohnungspolitische Transformationsprozesse eine grundlegende Veränderung der Eigentümerstruktur hervorgebracht, sodass zentrale Annahmen der Ertragslückentheorie im Gallus nun gültig sind.

\section{Abschaffung der Wohnungsgemeinnützigkeit}

Durch die Abschaffung des Wohnungsgemeinnützigkeitsgesetzes (WGG) durch die CDU/FDP-Regierung unter Helmut Kohl im Jahre 1990 haben in der Bundesrepublik schlagartig Millionen Wohnungen im Besitz gemeinnütziger Wohnungsunternehmen ihre gesetzlichen Preisbindungen verloren (Kuhnert/Leps 2017). Betroffen waren davon auch knapp 6o Prozent des Wohnungsbestandes im Gallus. Die Deregulierung des ehemals gemeinnützigen Sektors hat den Weg dafür bereitet, dass in den folgenden Jahren weite Teile der Wohnraumversorgung gewinnorientiert restrukturiert, privatisiert und einer Finanzmarktlogik unterworfen worden sind.

- Gewinnorientierte Neuausrichtung öffentlicher Wohnungsunternehmen: Nach Abschaffung der gesetzlichen Preisbindungen haben politische Entscheidungsträger_innen in den Stadt- und Landesregierungen ihre neu gewonnene Flexibilität genutzt, um die drei öffentlichen Wohnungsunternehmen gewinnorientiert zu restrukturieren. Bezogen auf die städtische ABG Frankfurt Holding, die heute im Gallus mit 5.500 Wohneinheiten knapp 30 Prozent des Angebots kontrolliert, bedeutet dies beispielsweise, dass das Unternehmen seit Anfang der 1990er Jahre den Wohnungsbestand umfangreich modernisiert, die Mieten an das ortsübliche Marktniveau angepasst, sich aus dem geförderten Wohnungsbau zurückgezogen und im Gallus selbstbewusst als Speerspitze der Aufwertung und Gentrifizierung agiert hat (Schipper/Wiegand 2015: 22ff.). Obschon das städtische Wohnungsunternehmen auch nach Abschaffung der Preisbindung laut Gesellschaftervertrag eigentlich weiterhin auf eine „sozial verantwortbare Wohnungsversorgung der breiten Schichten der Bevölkerung“ (ABG Frankfurt Holding 1997: 3) 
verpflichtet ist, begreift sich die ABG seit den 1990er Jahren als „ein Wirtschaftsunternehmen“, das unter der Maxime agiert, nur „etwas [zu] machen“, wenn „wir damit Geld verdienen“ (Geschäftsführer Frank Junker zit. n. Schipper/Wiegand 2015: 24). Gleiches gilt ebenso für die beiden landeseigenen Wohnungsunternehmen. Auch die Nassauische Heimstätte (NH) und die GWH Wohnungsbaugesellschaft im Besitz der Landesbank Hessen-Thüringen (Helaba) unterscheiden sich seit Abschaffung der Gemeinnützigkeit trotz ihrer öffentlichen Eigentümerschaft in ihrer Geschäftspraxis kaum (NH) bis gar nicht (GWH) von profitorientierten privaten Akteuren. Im Gallus folgt daraus, dass die öffentlichen Unternehmen als Motoren der Gentrifizierung agieren und Verdrängungsprozesse antreiben statt sie abzumildern (Schipper/ Wiegand 2015). Inwiefern aktuell zumindest bei der städtischen ABG ein Umdenken zu diagnostizieren ist, werden wir im Abschnitt 4 diskutieren.

- Privatisierung und Finanzialisierung ehemals gemeinnütziger Wohnungsbestände: Mit dem Wegfall der Preisbindungen sind die Wohnungsbestände zudem attraktiv geworden für gewinnorientierte Immobilieninvestoren. In den Fällen, in denen die ehemals gemeinnützigen Wohnungsanbieter nicht selbst die profitabelste Verwertung ihrer Wohngebäude durchsetzen konnten oder wollten, stand daher häufig der Verkauf beziehungsweise die Privatisierung der Bestände an institutionelle Investoren an (Unger 2016). Im Gallus ist es vor allem die Vonovia, die die ehemals preisgebundenen Wohnkomplexe der Deutschen Bundesbahn mit Bestandsmieten von um die fünf Euro pro Quadratmeter aufgekauft und als börsennotiertes Unternehmen einer Finanzmarktlogik unterworfen hat. Was dies für Mieter_innen bedeutet, lässt sich am eindrücklichsten in den Arbeitersiedlungen der Wallauer Straße sowie der Knorrstraße beobachten, die in den 1950er Jahren mit Fördermitteln des sozialen Wohnungsbaus errichtet worden sind und die nun in unmittelbarer Nachbarschaft zum gehobenen Europaviertel liegen (Abb. 3). Dort hat die Vonovia unter erheblichem Protest der Mieter_innen[4] eine Nachverdichtung „zu marktüblichen Mieten“ von ca. 13 bis 14 Euro pro Quatdratmeter sowie umfangreiche Modernisierungsmaßnahmen in Höhe von mehreren zehn Millionen Euro angekündigt, um architektonisch „die ästhetische Lücke zum Europaviertel“[5] zu schließen. Gelingt es der Vonovia, ihre Modernisierungsvorhaben wie geplant umzusetzen, drohen den bisherigen Mieter_innen drastische Mieterhöhungen von mehreren hundert Euro pro Monat, was für viele in der direkten Verdrängung und dem Verlust ihrer Nachbarschaft münden würde.

- Genossenschaften und Stiftungen: Allerdings gibt es auch im Gallus noch Nischen einer nicht-gewinnorientierten Wohnungswirtschaft (Abb. 2). Drei Traditionsgenossenschaften sowie das St. Katharinen- und Weißfrauenstift und die Stiftung Waisenhaus verfügen im Stadtviertel weiterhin über Wohnraum, den sie auch ohne gesetzliche Verpflichtung freiwillig nach den Prinzipien der Gemeinnützigkeit bewirtschaften. Deren Mieter_innen sind daher auch heute noch vor drastischen Mietsteigerungen und Verdrängung geschützt. In vielen Teilen des Arbeiterviertels wird die Eigentümerstruktur jedoch gegenwärtig - in deutlichem 
Kontrast zu den 1970er Jahren (Abb. 1) - von institutionellen Investoren und gewinnorientierten öffentlichen Wohnungsunternehmen dominiert (Abb.2), welche wachsende Ertragslücken nutzen und Mieten konsequent erhöhen.

Privatisierung öffentlicher Liegenschaften: Neubau-Gentrifizierung im Europaviertel

Seitdem die Deutsche Bahn 1998 den Hauptgüterbahnhof in Frankfurt aufgegeben hat, wird in nördlicher Nachbarschaft zum Gallus (Abb. 3) auf einer Fläche von rund 145 Hektar und mit einem Investitionsvolumen von mehreren Milliarden Euro das Europaviertel errichtet. Nach seiner Fertigstellung Anfang der 2020er Jahre soll es neben 30.000 Arbeitsplätzen und Erweiterungsflächen für die Messe auch Platz für 6.00o Wohnungen bieten. Weil jedoch erstens die Deutsche Bahn ihre öffentliche Liegenschaft zum Höchstgebot an die Immobilienfirmen CA Immo und Aurelis Real Estate privatisiert hat, zweitens die Stadt Frankfurt ihre stadtplanerischen Instrumente kaum genutzt hat, um Investoren etwa zum Bau von gefördertem und sozialem Wohnraum zu zwingen, und drittens auch die städtischen und landeseigenen Wohnungsbauunternehmen mit Mietwohnungen ab zwölf Euro Kaltmiete pro Quadratmeter und Eigentumswohnungen für Quadratmeterpreise bis zu 10.00o Euro lediglich hochpreisige Segmente bedienen, konnte nördlich des alten Gallus fast ausschließlich gehobener und luxuriöser Wohnraum für eine zahlungskräftige Klientel entstehen (Schipper/Wiegand 2015).

Bemerkenswert sind die kleinräumigen Preisdifferenzen zwischen den Neubauarealen im Europaviertel und dem alten Wohnungsbestand im Gallus. Gemäß dem IHK Wohnungsmarktbericht lagen 2013 die Kaltmieten bei Neuvertragsabschlüssen im Europaviertel um 40 Prozent und die Eigentumspreise um 70 Prozent über denjenigen im alten Gallus (ebd: 22). Diese erheblichen nahräumlichen Ertragslücken deuten auf eine drohende NeubauGentrifizierung hin (Stabrowski 2014). Im Sinne der Rent-Gap-Theorie kann das gegenwärtig erzielte Mietniveau im Europaviertel aufgrund der gleichen Lagequalitäten zugleich als Annäherung an die potenzielle Grundrente im gesamten Gallus gelesen werden. Denn aus Sicht privater Investoren gibt es keinen Grund, warum nach Aufwertung, Modernisierung oder Abriss die Mieten im jetzigen Bestand dauerhaft niedriger sein sollen als diejenigen, die sich auf den Neubauflächen nebenan realisieren lassen. Die Modernisierungspläne der Vonovia offenbaren diesbezüglich, dass ein solcher Verdrängungsprozess bereits begonnen hat. Der Ausstrahlungseffekt steigender Mieten wird zudem auch indirekt durch die Funktionsweise des Mietspiegels befördert. Da die hohen Mietabschlüsse im Europaviertel in die Berechnung der ortsüblichen Vergleichsmiete einfließen, werden zukünftig erhebliche Mieterhöhungen im Bestand möglich sein (Schipper/Wiegand 2015).

Da es sich beim ehemaligen Güterbahnhof um eine öffentliche Liegenschaft gehandelt hat und zudem das deutsche Planungsrecht verschiedene Instrumente bietet, Investoren zum Bau von preisgünstigem Wohnraum zu zwingen (Abschnitt 2.2), hätte der Prozess der Neubau-Gentrifizierung verhindert oder zumindest abgemildert werden können - wenn es den dafür notwendigen politischen Willen gegeben hätte. 
Investorenfreundliche Stadtplanung: Schaffung von Baurecht für gehobenen Wohnraum

Dass sich südlich des Europaviertels bei den umfassenden Neubautätigkeiten auf ehemaligen Industriebrachen im alten Gallus gleichfalls weitgehend überall die profitabelste Nutzung durchsetzen konnte, ist ebenfalls maßgeblich auf politische Entscheidungen zurückzuführen. Um einkommensstarke Bevölkerungsgruppen, die seit den 1980er Jahren zunehmend ins Umland gezogen sind, wieder nach Frankfurt zu locken, hat die Stadt ab Anfang der 1990er Jahre gezielt Aufwertungsprozesse angestoßen. Private Investoren sollten „mit den geringsten Regulierungskosten und den geringsten planerischen Restriktionen“ (Stadtplanungsdezernent Martin Wentz, zit. n. Silomon-Pflug 2018: 263) zur Realisierung hochpreisigen Wohnraums motiviert werden. Die Auswirkungen einer solchen investorenfreundlichen Stadtentwicklung haben sich mittlerweile im Gallus materialisiert. Auf den zu Wohnbauland umgewidmeten ehemaligen Industriebrachen (Abb. 3) haben verschiedene Immobilienentwickler insgesamt knapp 2.00o Wohnungen im überwiegend gehobenen Segment realisieren können, weil die Stadt konsequent und bewusst auf die Anwendung stadtplanerischer Instrumente zur Schaffung preisgünstigen Wohnraums verzichtet hat.

Zusammenfassend kann die Rent-Gap-Theorie den aktuellen Gentrifizierungsdruck im Gallus erklären. Allerdings führen die wachsenden Differenzen zwischen gegenwärtiger und maximal möglicher Verwertung nicht automatisch zu Mietsteigerungen und Verdrängung. Möglich wird dies nur, weil politische Akteure auf verschiedenen staatlichen Ebenen seit Anfang der 1990er Jahre die zentralen Gültigkeitsbedingungen der Ertragslückentheorie wahr gemacht haben - und zwar konkret durch die Abschaffung der Wohnungsgemeinnützigkeit, die anschließende gewinnorientierte Neuausrichtung öffentlicher Wohnungsunternehmen, die Privatisierung ehemals gemeinnütziger Wohnungsbestände und öffentlicher Liegenschaften sowie eine ausgewiesen investorenfreundliche Stadtplanung, die Prozesse der Neubau-Gentrifizierung induziert hat.

\section{Ausblick: „How to make rent gap theory untrue again?“}

Gentrifizierung stellt somit eine sozialräumliche Restrukturierung der urbanen Klassenstruktur dar, bei der politische Akteure über erhebliche Handlungsspielräume und Einflussmöglichkeiten verfügen. Mietrechtliche Regularien, stadtplanerische Entscheidungen und die jeweilige Eigentümerstruktur entscheiden darüber, inwiefern sich ein immobilienwirtschaftlicher Verwertungsdruck auch in Verdrängungsprozesse übersetzt. Damit die Rent-Gap-Theorie keine Gültigkeit mehr beanspruchen kann und einkommensschwache Haushalte auch in Vierteln unter hohem Gentrifizierungsdruck effektiv vor Verdrängung geschützt sind, können Kommunen über stadtplanerische Instrumente und eine sozialgerechte Liegenschaftspolitik Einfluss auf das Preisniveau von Neubauprojekten nehmen sowie ihre öffentlichen Wohnungsunternehmen wieder auf eine gemeinnützige Bewirtschaftungspraxis verpflichten. Dort, wo hingegen private Eigentümer_innen und institutionelle Investoren ein Stadtviertel dominieren, haben Stadtregierungen kaum Einfluss. Hier lassen sich 


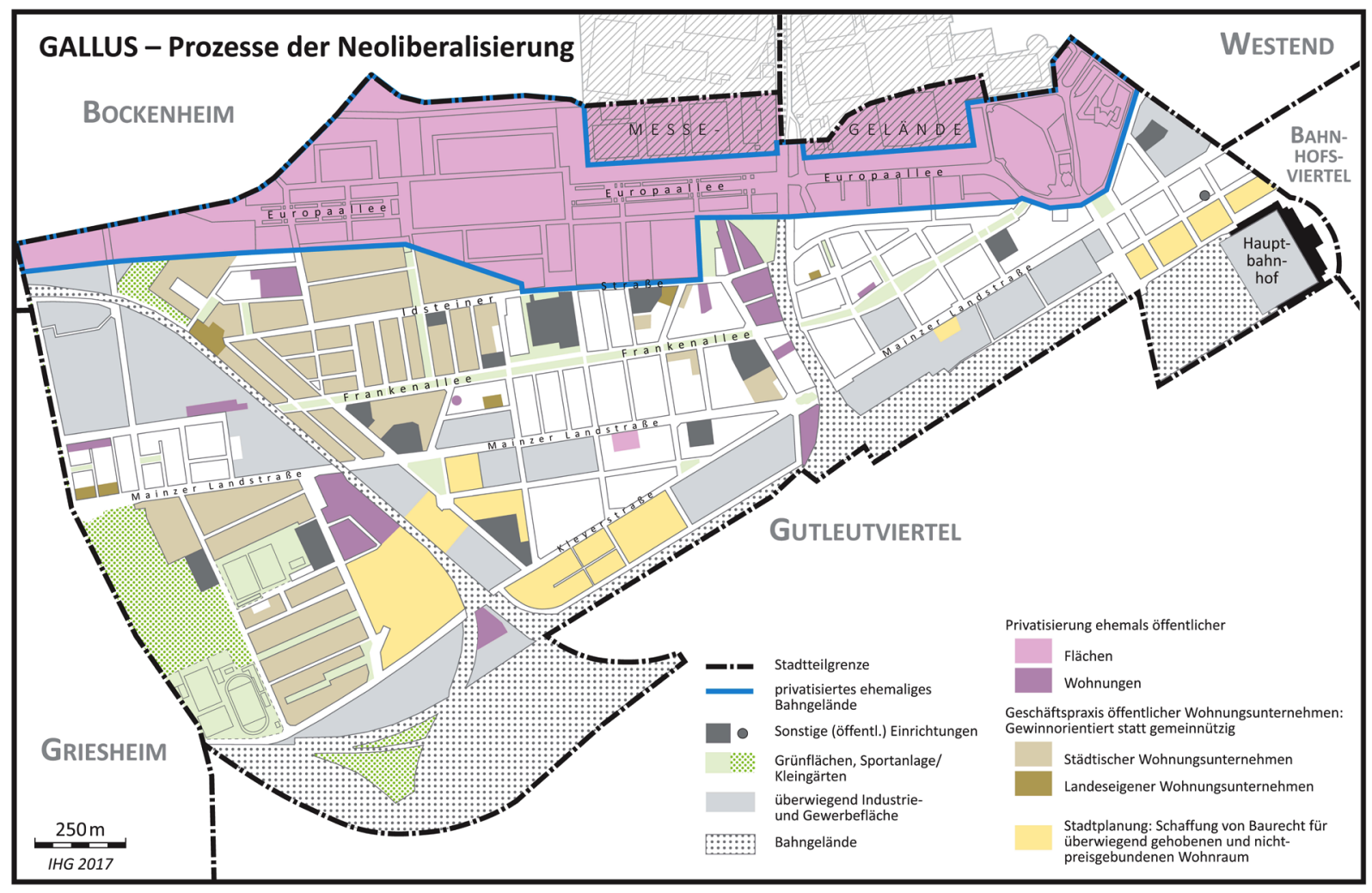

Verdrängungsprozesse aber über eine Verschärfung des Mietrechts auf Abb. 3 Prozesse der Bundes- und Landesebene abwenden. Bezogen auf das Gallus sind die skalenspezifischen Potenziale für eine postneoliberale Wohnungspolitik in Abbildung 4 dargestellt. Neoliberalisierung im Frankfurter Gallus seit 1990

\subsection{Stadtplanerische Instrumente konsequent anwenden (Stadt)}

Da die großen öffentlichen Grundstücke im Gallus bereits privatisiert worden sind, hat die Stadt Frankfurt über ihre Liegenschaftspolitik keine direkte Möglichkeit mehr, bezahlbaren Wohnraum sicherzustellen. Allerdings könnte die Kommune bezogen auf die verbliebenen Konversionsflächen in privatem Eigentum ihre stadtplanerischen Instrumente nutzen, um Investoren über städtebauliche Verträge zur Schaffung von preisgebundenem und genossenschaftlichem Wohnraum zu zwingen. Wie aus Abbildung 4 hervorgeht, existieren im Gallus jedoch mittlerweile nur noch relativ wenige industrielle Brachflächen, auf denen die Stadt noch derart steuernd eingreifen könnte. Wesentliche Weichenstellungen sind in der Vergangenheit schon zugunsten von hochpreisigem Wohnraum getroffen worden (Abb. 3).

\section{2. Öffentliche Wohnungsunternehmen nicht-gewinnorientiert ausrichten (Stadt und Land)}

Da die öffentlichen Wohnungsbestände im Gallus in weiten Teilen nicht privatisiert worden sind, können Stadt und Land über ihre Wohnungsunternehmen weiterhin direkten Einfluss auf Mietpreisniveaus und Verdrängungsprozesse ausüben. Allein die städtische Wohnungsbaugesellschaft ABG Frankfurt Holding verfügt mit 5.500 Wohneinheiten über einen Marktanteil von circa 30 Prozent aller Mietwohnungen im Gallus (Schipper/ Wiegand 2015: 23). Hinzu kommen weitere Bestände der landeseigenen 


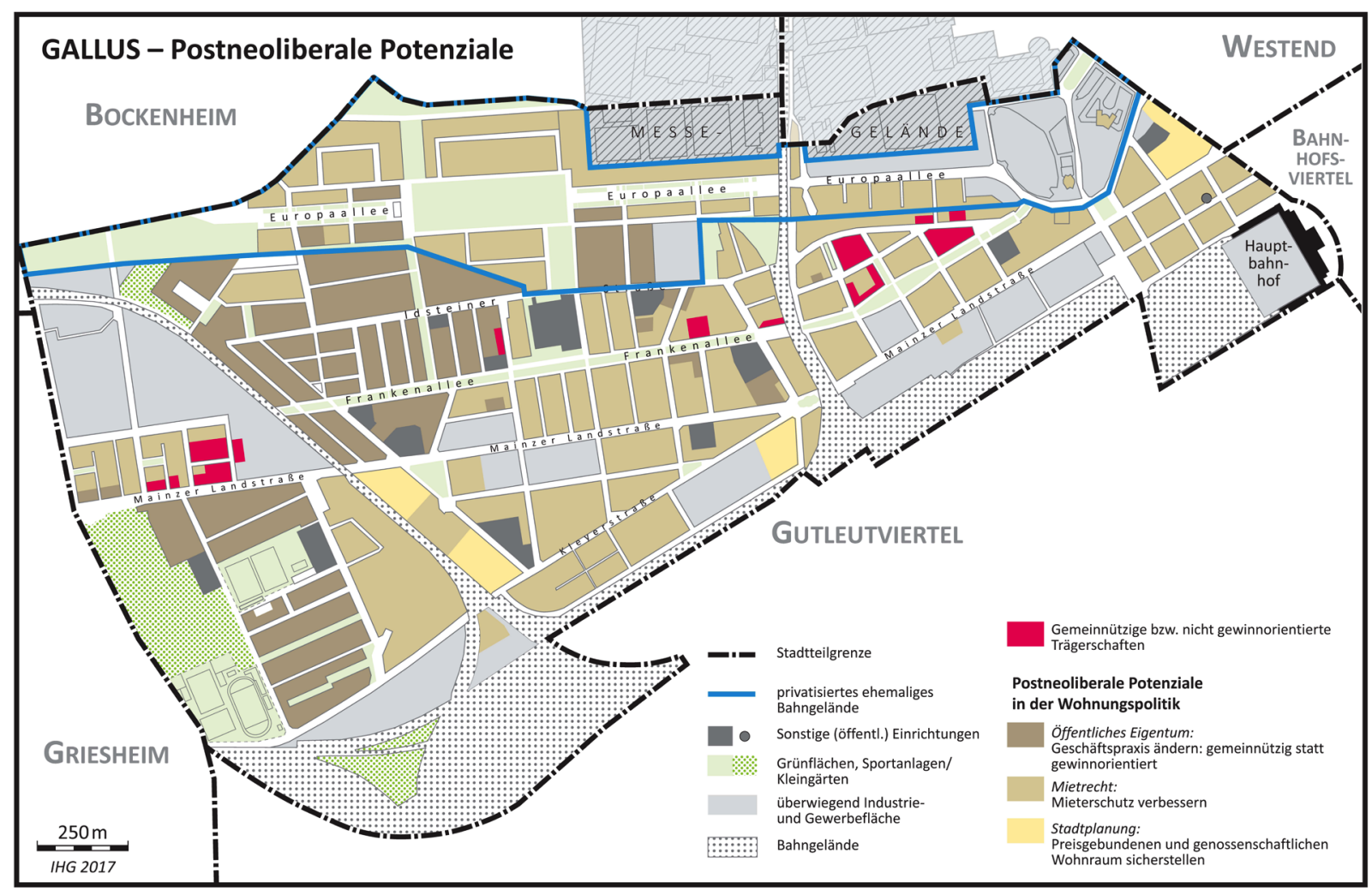

Abb. 4 Postneoliberale Potenziale zur Verhinderung von Verdrängung im Gallus
Nassauischen Heimstätte und der GWH (Abb. 2 und 4). Da alle drei Unternehmen jährlich hohe Überschüsse von jeweils bis zu 9o Millionen Euro erwirtschaften (Schipper 2018: 91), verfügen sie über beträchtliche ökonomische Handlungsspielräume zur Umsetzung einer moderaten Mietenpolitik, die Mieter_innen effektiv vor Verdrängung schützt. Aus diesem Grund haben Frankfurter Mieterinitiativen seit 2014 vehement einen Mietenstopp bei der ABG Holding eingefordert (Schipper 2018: 92ff.). Die Proteste waren insofern erfolgreich, als dass die Stadt Frankfurt auf Druck der SPD im Mai $2016 \mathrm{ihr}$ Wohnungsunternehmen darauf verpflichtet hat, die Bestandsmieten nur noch um maximal ein Prozent pro Jahr anzuheben. Einen ähnlichen Beschluss auch für die landeseigenen Wohnungsbaugesellschaften $\mathrm{NH}$ und GWH zu übernehmen, wie von Mieterinitiativen gefordert, wird allerdings bislang von der schwarz-grünen Landesregierung abgelehnt. Dennoch zeigt das Beispiel der ABG, dass die Gültigkeitsbedingungen der Rent-Gap-Theorie durch politische Entscheidungen aufgehoben werden können. Zumindest knapp ein Drittel der Mieterhaushalte im Gallus sind dank des erkämpften Mietenstopps zukünftig vor Verdrängung geschützt.

\subsection{Neue Wohnungsgemeinnützigkeit einführen (Bund)}

Um derartige Erfolge wohnungspolitischer Initiativen auch langfristig abzusichern und in den öffentlichen Unternehmen eine sozial ausgerichtete Geschäftspraxis institutionell zu verankern, fordern das bundesweite Netzwerk „Mieten und Wohnen“[6] sowie zahlreiche weitere Akteure, die 1990 auf Bundesebene abgeschaffte Wohnungsgemeinnützigkeit in modifizierter Form wieder einzuführen (Schönig/Kadi/Schipper 2017). Kernanliegen einer solchen neuen Gemeinnützigkeit im Wohnungssektor wäre es, „eine strikte Non-Profit-Orientierung in der Bewirtschaftung, eine klar definierte Zweckbindung der unternehmerischenZiele sowie [...] eine effektivegesellschaftliche 
Kontrolle“(Holm/Horlitz/Jensen 2015: 41) gesetzlich festzuschreiben. Demgemäß müssten gemeinnützige Wohnungsunternehmen ihre Mieten auf Grundlage des Kostendeckungsprinzips kalkulieren, die Eigenkapitalrendite begrenzen, ihre Zielgruppe auf mittlere und niedrige Einkommen beschränken sowie demokratische Verfahren der Mietermitbestimmung etablieren (Kuhnert/Leps 2017). Im Gegenzug bekämen die Träger_innen, die sich freiwillig den Prinzipien der Gemeinnützigkeit verpflichten, Steuervergünstigungen sowie einen privilegierten Zugang zu öffentlichen Fördergeldern und Bauflächen. Auf einer solchen gesetzlichen Grundlage könnten die Stadt Frankfurt und das Land Hessen ihre Wohnungsunternehmen gemeinnützig ausrichten und dadurch zu Vorreitern einer neuen nicht-gewinnorientierten Wohnungswirtschaft werden. Mieter_innen in öffentlichen Wohnungsunternehmen wären so effektiv und langfristig vor Verdrängung geschützt.

\subsection{Mietrecht verschärfen (Bund)}

Der Großteil der Wohnraumversorgung im Gallus liegt jedoch angesichts der Neoliberalisierungsprozesse seit Anfang der 1990er Jahre mittlerweile in der Hand privater Eigentümer_innen und institutioneller Investoren (Abb. 2 und 4). Der Einfluss kommunaler Wohnungspolitik ist hier marginal. Allerdings lassen sich Verdrängungsprozesse auch im privaten Bestand verhindern, indem Renditepotenziale gesetzlich begrenzt und mietrechtliche Regularien im Interesse der Mieter_innen verschärft werden. Die gesetzgeberischen Kompetenzen sind jedoch auf Bundes- und zum Teil auf Landesebene verortet. Beispielsweise könnte die Bundesregierung alle Bestandsmieten in die Bestimmung der ortsüblichen Vergleichsmiete einbeziehen, die Modernisierungsumlage abschaffen und die Kappungsgrenze für Mieterhöhungen bei bestehenden Mietverträgen von derzeit 15 Prozent in drei Jahren weiter absenken. Bezüglich der Wiedervermietung von Wohnraum wäre darüber hinaus die bislang kaum wirksame Mietpreisbremse grundlegend zu überarbeiten, indem Ausnahmeregelungen reduziert werden und ihre reale juristische Durchsetzbarkeit für Mieter_innen erleichtert wird. Bei entsprechenden parlamentarischen Mehrheitsverhältnissen ließen sich solche Vorhaben auch gegen die Interessen und den Widerstand der Immobilienwirtschaft politisch umsetzen. Eine derartige Regulation des Mietmarktes, die die Gültigkeitsbedingungen der Rent-Gap-Theorie praktisch aufhebt, wäre zudem auch ökonomisch realisierbar. Selbst eine strenge Wohnraumzwangsbewirtschaftung, die Mieterhöhungen jenseits der Inflationsrate völlig unterbände, würde das investierte Baukapital nicht entwerten und die Bausubstanz nicht gefährden (Brede/Kohaupt/ Kujath 1975: 73). Verhindert würden lediglich Extraprofite, die derzeit bei angespannten Wohnungsmärkten durch die Möglichkeit entstehen, sich aufgrund der Knappheit preisgünstigen Wohnraums privat eine höhere Grundrente anzueignen.

\subsection{Kollektive Mieterrechte etablieren (Bund)}

Bezüglich der profitorientierten, institutionellen Investoren, dieim Gallus mittlerweile einen bedeutenden Bestand der Wohnraumversorgung kontrollieren 
(Abb. 2), ist eine Verbesserung der individuellen Rechtsstellung der Mieter_innen aber womöglich nicht ausreichend, da jeder einzelne Haushalt seine Rechte allein gegen mächtige und finanzstarke Akteure einklagen müsste. Hilfreich wäre es daher, finanzmarktorientierte Wohnungskonzerne wie Vonovia einer strengen gesellschaftlichen Kontrolle zu unterwerfen. Knut Unger (2017) vom Mieterverein Witten schlägt diesbezüglich ein spezielles Wohnungswirtschaftsrecht vor, welches die Mieterschaft der Großvermieter zu Rechtssubjekten mit kollektiven Rechten und einem Verbandsklagerecht erklärt sowie Instandhaltungspraktiken, Mieterhöhungen und Betriebskostenabrechnungen einer besonderen Regulation unterwirft. Würde durch eine solche Gesetzgebung Wohnraum als Kapitalanlage für renditeorientierte Finanzinvestitionen unattraktiver, könnte zudem ein Ausstieg aus der finanzmarktdominierten Wohnungswirtschaft und eine schleichende Veränderung der Eigentümerstruktur zugunsten nicht-gewinnorientierter Akteure eingeleitet werden. Aus Sicht von Mieter_innen in Stadtvierteln wie dem Gallus wäre eine solche Transformation wünschenswert, weil dadurch die strukturellen Gültigkeitsbedingungen der Rent-Gap-Theorie ähnlich zur Situation in den 1970er Jahren (Abb. 1) wieder aufgehoben würden und man vor Verdrängung geschützt wäre.

Zusammenfassend lassen sich Verdrängungsprozesse durch eine sozialgerechte Stadtplanung, eine Stärkung nicht-profitorientierter Wohnungsunternehmen und eine grundlegende Reform des Mietrechts verhindern. Politische Bestrebungen, die darauf abzielen, die Gültigkeitsbedingungen der Rent-Gap-Theorie im Interesse von Mieter_innen aufzuheben, sind allerdings erfahrungsgemäß mit erheblichem Gegenwind aus staatlichen Apparaten und den öffentlichen Wohnungsunternehmen konfrontiert (Schipper 2018; Vogelpohl et al. 2017). Da sich mit Immobilien insbesondere in von Gentrifizierung bedrohten Stadtteilen hohe Renditen erwirtschaften lassen, ist ebenso der Widerstand der gewinnorientierten Wohnungswirtschaft und ihrer Lobbyverbände enorm. Die von Eric Clark aufgeworfene Frage: „[H]ow might urban governance contribute to making rent gap theory not true?" (Clark 2014) ist daher nicht nur auf ökonomischer, technischer und planerischer Ebene zu beantworten. Sie ist vor allem eine Frage gesellschaftlicher Machtverhältnisse und politischer Mehrheiten auf allen staatlichen Ebenen.

\section{Endnoten}

[1] Dank für die Erstellung der Karten gilt Elke Alban.

[2] Mit ca. 355.00o Wohneinheiten ist die Vonovia das größte Wohnungsunternehmen in Deutschland.

[3] http://www.deutsches-architektur-forum.de/forum/forumdisplay.php?f=170 (letzter Zugriff am 7.4.2018).

[4] http://www.fr.de/frankfurt/wohnen/mieten-in-frankfurt-gallus-leidet-untereuropaviertel-a-1265543 (letzter Zugriff am 9.4.2018).

[5] http://www.deal-magazin.com/news/3/62623/Vonovia-investiert-134-Mio-Euro-inKnorr-Quartier-in-Frankfurt (letzter Zugriff am 9.4.2018).

[6] http://www.netzwerk-mieten-wohnen.de/ (letzter Zugriff am 9.4.2018). 


\section{Autor_innen}

Sebastian Schippers Forschungsschwerpunkte beinhalten Stadtpolitik, politische Ökonomie des Wohnens, Gentrifizierung und städtische soziale Bewegungen.

s.schipper@geo.uni-frankfurt.de

Tabea Latocha beschäftigt sich mit der Urban Citizenship Debatte und der Rolle der Bürger_ innen in städtischen Planungsprozessen.

tabea.latocha@live.de

Die Publikation dieses Beitrags wurde durch die Deutsche Forschungsgemeinschaft (DFG) und den Open-Access-Fonds der Goethe-Universität Frankfurt ermöglicht.

\section{Literatur}

ABG Frankfurt Holding (1997): Gesellschaftsvertrag der ABG Frankfurt-Holding Wohnungsbau- und Beteiligungsgesellschaft mbH. Frankfurt am Main.

Atkinson, Rowland (2015): Losing one's place. Narratives of neighbourhood change, market injustice and symbolic displacement. In: Housing, Theory and Society 32/4, 373-388.

Balmer, Ivo / Bernet, Tobis (2017): Selbstverwaltet bezahlbar wohnen? Potentiale und Herausforderungen genossenschaftlicher Wohnprojekte. In: Barbara Schönig / Justin Kadi / Sebastian Schipper (Hg.), Wohnraum für alle?! Perspektiven auf Planung, Politik und Architektur. Bielefeld: transcript, 259-280.

Blasius, Jörg (2004): Gentrification und die Verdrängung der Wohnbevölkerung. In: Robert Kecskes / Michael Wagner / Christof Wolf (Hg.), Angewandte Soziologie. Wiesbaden: VS Verlag, 21-44.

Brede, Helmut / Kohaupt, Bernhard / Kujath, Hans-Joachim (1975): Ökonomische und politische Determinanten der Wohnungsversorgung. Frankfurt am Main: Suhrkamp Verlag.

Clark, Eric (1995): The rent gap re-examined. In: Urban Studies 32/9, 1489-1503.

Clark, Eric (2014): Good urban Governance: Making rent gap theory not true. In: Geografiska Annaler: Series B, Human Geography 96/4, 392-395.

Davidson, Mark / Lees, Loretta (2005): New-build 'gentrification' and London's riverside renaissance. In: Environment and Planning a 37/7, 1165-1190.

Fields, Desiree / Uffer, Sabine (2016): The financialisation of rental housing. A comparative analysis of New York City and Berlin. In: Urban Studies 53/7, 1486-1502.

Frank, Susanne (2017): Gentrifizierung und neue Mittelschichten: Drei Phasen eines wechselhaften Verhältnisses. In: Barbara Schönig / Justin Kadi / Sebastian Schipper (Hg.), Wohnraum für alle?! Perspektiven auf Planung, Politik und Architektur. Bielefeld: transcript, 87-100.

Friedrichs, Jürgen / Blasius, Jörg (2016): Die Kölner Gentriciation-Studien. In: Jürgen Friedrichs / Jörg Blasius (Hg.), Gentrifizierung in Köln. Soziale, ökonomische, funktionale und symbolische Aufwertungen. Leverkusen-Opladen: Budrich Barbara, 7-28.

Harvey, David (2006 [1982]): The Limits to Capital. London: Verso.

Helbrecht, Ilse (1996): Die Wiederkehr der Innenstädte. Zur Rolle von Kultur, Kapital und Konsum im Prozeß der Gentrification. In: Geographische Zeitschrift 84/1, 1-15.

Helbrecht, Ilse / Weber-Newth, Francesca (2017): Die Abschöpfung des Planungsmehrwerts als Repolitisierung der Planung? In: sub \urban. zeitschrift für kritische stadtforschung $5 / 1 / 2,61-86$.

Holm, Andrej (2010): Wir bleiben alle! Gentrifizierung - Städtische Konflikte um Aufwertung und Verdrängung. Münster.

Holm, Andrej / Horlitz, Sabine / Jensen, Inga (2015): Neue Gemeinnützigkeit. Gemeinwohlorientierung in der Wohnungsversorgung. https://www.heidrun-bluhm.de/fileadmin/kreise/Bluhm/Neue_Gemeinnuetzigkeit_ gesamt_2015-09-16.pdf (letzter Zugriff am 9.2.2016).

Kuhnert, Jan / Leps, Olof (2017): Neue Wohnungsgemeinnützigkeit: Wege zu langfristig preiswertem und zukunftsgerechtem Wohnraum. Wiesbaden: Springer VS.

Lees, Loretta / Slater, Tom / Wyly, Elvin K. (2008): Gentrification. New York, NY: Routledge. 
Ley, David (1996): The New Middle Class and the Remaking of the Central City. Oxford: University Press.

Marcuse, Peter (1986): Abandonment, gentrification, and displacement: The linkages in New York City. In: Neil Smith / Peter Williams (Hg.), Gentrification of the City. Boston: Allen \& Unwin, 153-177.

Marx, Karl (1988 [1893]): Das Kapital. Kritik der politischen Ökonomie, Band 3. Der Gesamtprozeß der kapitalistischen Produktion. Berlin.

Mösgen, Andrea / Schipper, Sebastian (2017): Gentrifizierungsprozesse im Frankfurter Ostend. Stadtpolitische Aufwertungsstrategien und Zuzug der Europäischen Zentralbank. In: Raumforschung und Raumordnung 75/2, 125-141.

Ronneberger, Klaus / Keil, Roger (1995): Ausser Atem - Frankfurt nach der Postmoderne. In: Hansruedi Hitz / Ute Lehrer / Roger Keil (Hg.), Capitales Fatales. Urbanisierung und Politik in den Finanzmetropolen Frankfurt und Zürich. Zürich, 286-353.

Schipper, Sebastian (2013a): Genealogie und Gegenwart der unternehmerischen Stadt. Neoliberales Regieren in Frankfurt am Main, 1960-2010. Münster: Westfälisches Dampfboot.

Schipper, Sebastian (2013b): Global-City-Formierung, Gentrifizierung und Grundrentenbildung in Frankfurt am Main. In: Zeitschrift Für Wirtschaftsgeographie 57/4, 185-200.

Schipper, Sebastian (2018): Wohnraum dem Markt entziehen? Wohnungspolitik und städtische soziale Bewegungen in Frankfurt und Tel Aviv. Wiesbaden: Springer VS.

Schipper,Sebastian/Wiegand,Felix(2015): Neubau-Gentrifizierungund globale Finanzkrise. Der Stadtteil Gallus in Frankfurt am Main zwischen immobilienwirtschaftlichen Verwertungszyklen, stadtpolitischen Aufwertungsstrategien und sozialer Verdrängung. In: sub\ urban. zeitschrift für kritische stadtforschung 3/3, 7-32.

Schönig, Barbara / Kadi, Justin / Schipper, Sebastian (Hg.) (2017): Wohnraum für alle?! Perspektiven auf Planung, Politik und Architektur. Bielefeld: transcript.

Silomon-Pflug, Felix (2018): Verwaltung der unternehmerischen Stadt. Zur neoliberalen Neuordnung von Liegenschaftspolitik und -verwaltung in Berlin und Frankfurt am Main. Bielefeld: transcript.

Slater, Tom (2017): Planetary Rent Gaps. In: Antipode 49/1, 114-137.

Smith, Neil (1979): Toward a theory of gentrification. A back to the city movement by capital, not people. In: Journal of the American Planning Association 45/4, 538-548.

Smith, Neil (1996): The new urban frontier. Gentrification and the revanchist city. London: Psychology Press.

Sozialwissenschaftliche Arbeitsgruppe Mühlich \& Mühlich (1974): Sozialwissenschaftliche Problemanalyse zur Strukturplanung Gallus. Frankfurt am Main (unveröffentlichte Studie).

Stabrowski, Filip (2014): New-build gentrification and the everyday displacement of Polish immigrant tenants in greenpoint, Brooklyn. In: Antipode 46/3, 794-815.

Unger, Knut (2016): Financialization of mass rental housing in Germany: Understanding the transaction cycles in the mass rental housing sector 1999-2015. In: Barbara Schönig / Sebastian Schipper (Hg.), Urban Austerity: Impacts of the Global Financial Crisis on Cities in Europe. Berlin: Theater der Zeit, 176-190.

Unger, Knut (2017): So bändigen wir die Wohnungsriesen. Ansätze zur gesellschaftlichen Kontrolle der finanzmarktorientierten Vermietungskonzerne. https://www.rosalux. de/fileadmin/rls_uploads/pdfs/Standpunkte/Standpunkte_11-2017_web.pdf (letzter Zugriff am 15.9.2017).

Vogelpohl, Anne / Vollmer, Lisa / Vittu, Elodie / Norma, Brecht (2017): Die Repolitisierung des Wohnens. Städtische soziale Bewegungen für ein Recht auf Wohnen und auf Stadt in Hamburg, Berlin, Jena und Leipzig. In: Barbara Schönig / Justin Kadi / Sebastian Schipper (Hg.), Wohnraum für alle?! Perspektiven auf Planung, Politik und Architektur. Bielefeld: transcript, 105-130. 


\section{How to stop displacement? The rent gap theory of gentrifi- cation and its validity taking the Gallus in Frankfurt as an example}

Within the broad literature on gentrification, the rent gap theory analyzes how gaps between actual and potential ground rents cause the displacement of working class households. In contrast, Eric Clark (2014) recently raised the inverted question "how might urban governance contribute to making rent gap theory not true?" Regarding the German context and taking the Gallus neighborhood in Frankfurt as an example, we demonstrate that rent regulation, urban planning policies and the specific structure of the land ownership determine whether the emergence of a rent gap actually leads to the displacement of working class people. Therefore, gentrification is not a law of nature, but a deeply political process that can be stopped and prevented effectively - given a strong political commitment to do so. 